\title{
Cystic pancreatic lesions: MR imaging findings and management
}

\author{
Giovanni Morana ${ }^{1 *} \mathbb{D}$, Pierluigi Ciet ${ }^{2}$ and Silvia Venturini ${ }^{1}$
}

\begin{abstract}
Cystic pancreatic lesions (CPLs) are frequently casual findings in radiological examinations performed for other reasons in patients with unrelated symptoms. As they require different management according to their histological nature, differential diagnosis is essential. Radiologist plays a key role in the diagnosis and management of these lesions as imaging is able to correctly characterize most of them and thus address to a correct management. The first step for a correct characterization is to look for a communication between the CPLs and the main pancreatic duct, and then, it is essential to evaluate the morphology of the lesions. Age, sex and a history of previous pancreatic pathologies are important information to be used in the differential diagnosis. As some CPLs with different pathologic backgrounds can show the same morphological findings, differential diagnosis can be difficult, and thus, the final diagnosis can require other techniques, such as endoscopic ultrasound, endoscopic ultrasound-fine needle aspiration and endoscopic ultrasound-through the needle biopsy, and multidisciplinary management is important for a correct management.
\end{abstract}

Keywords: Pancreatic cyst, Pancreatic neoplasms, Pancreatic intraductal neoplasms, Cystadenoma, Serous, Cystadenoma, Mucinous

\section{Key points}

- CPLs are frequently detected lesions with different malignant potential, requiring different management.

- MRI with MRCP best allows detection of those imaging features helpful for lesion characterization.

- A CPL communicating with MPD is an IPMN.

- A CPL non-communicating with MPD is related to different pathologies (SCN, SPN, MCN).

- Differential diagnosis can be done using morphological appearance, location and demographic data.

*Correspondence: gmorana61@gmail.com; giovanni.morana@aulss2.veneto.it 1 Department of Radiology, Regional Hospital Ca'Foncello, Piazza Ospedale 1, 3100 Treviso, Italy

Full list of author information is available at the end of the article

\section{Introduction}

Cystic pancreatic lesions (CPLs) are quite common: Their frequency of detection ranges from 2.4 to $19.6 \%$, and their prevalence as well as size and number increases with age (from 7.9 below 70 years to 40.2 over 70 years) [1-5]. A precise characterization is fundamental for the correct management of these lesions, as they have heterogeneous biological behavior and different prognosis (according to histological type and differentiation), thus requiring different therapeutic options [6].

However, difficulties in differential diagnosis of CPLs still exist because of the lack of specific clinical and laboratoristic signs and the overlap of imaging findings, and thus, the management of patients with CPLs remains complex [7]. Moreover, the frequent incidental detection of CPLs, in the absence of any symptoms, makes the diagnosis even more difficult [8].

Most CPLs can be considered "technopathies," as they are more frequently detected in the last decades due to the widespread use and advancement in diagnostic 
imaging. The vast majority of these lesions will never threat the life of affected subjects, but due to their malignant potential will cause affected subjects to become patients, and followed up even for many years. Follow-up strategies rise a really challenging issue for radiologists, due to the high number of patients to be submitted. The role of Imaging is to differentiate benign from malignant or potentially malignant CPLs avoiding unnecessary surgery and, in potentially malignant CPLs, to early detect morphological changes related to malignant transformations in order to offer more chance of survival to these patients.

Scope of this review is to offer a practical approach to the diagnosis of CPLs using mainly MR imaging findings, location and demographic data and thus drive their correct management. Differential diagnosis among different CPLs is also emphasized.

\section{Classification}

According to data extrapolated from WHO classification, CPLs are classified in epithelial and non-epithelial and each of these categories is further subdivided in nonneoplastic and neoplastic (CPNs) (Table 1) [9]

Epithelial CPNs are further divided in mucinous, always premalignant-malignant lesions and non-mucinous neoplasms, which include both benign (most frequent) and borderline and malignant lesions.

In particular, mucinous CPNs include mucinous cystic neoplasm (MCN) and intraductal papillary mucinous neoplasm (IPMN), while non-mucinous CPNs include serous cystic neoplasm (SCN), solid pseudopapillary neoplasm (SPN), cystic neuroendocrine neoplasm (CPNET), the rarer acinar cell cystic neoplasm (ACCN), ductal adenocarcinoma with cystic degeneration and other rarer lesions (Table 1).

Many others CPLs can be included in this review, but due to their very low incidence, their discussion will be omitted [10].

From the radiological viewpoint, the key factor to characterize a CPL is to establish whether the lesion communicates or not with the main pancreatic duct (MPD) (Table 2).

CPLs non-communicating with MPD include nonneoplastic (walled-off necrosis-WON, simple or congenital cyst and retention cyst) as well as neoplastic cysts [11, 12]. Neoplastic non-communicating cysts include mucinous ( $\mathrm{MCN})$ and non-mucinous neoplasms (SCN, SPN, ACCN, CPNET and ductal adenocarcinoma with cystic degeneration). CPLs communicating with MPD are further divided in

Table 1 Classification of cystic pancreatic lesions (CPLs)

WHO classification of cystic pancreatic lesions

Epithelial non-neoplastic

Lymphoepithelial cyst

Mucinous non-neoplastic cyst

Enterogeneous cyst

Retention cyst/dysontogenetic cyst

Periampullary duodenal wall cyst

Endometrial cyst

Congenital cyst (in malformation syndromes)

\author{
Epithelial neoplastic \\ Mucinous \\ Intraductal papillary mucinous neoplasm \\ Mucinous cystic neoplasm \\ Non-mucinous \\ Serous cystic neoplasm \\ Solid pseudopapillary neoplasm \\ Cystic neuroendocrine tumor G1-2 \\ Acinar cell cystadenoma \\ Serous cystoadenocarcinoma \\ Cystic ductal adenocarcinoma \\ Cystic acinar cell carcinoma \\ Accessory-splenic epidermoid cyst \\ Cystic hamartoma \\ Cystic teratoma \\ Cystic pancreatoblastomas \\ Cystic metastatic epithelial neoplasm \\ Others
}

Non-epithelial neoplastic

Benign non-epithelial neoplasms (e.g., lymphangioma) 
Table 2 Radiological classification of cystic pancreatic lesions (CPLS)

Cystic pancreatic lesions: radiological classification

Non-communicating with main pancreatic duct

Non-neoplastic

Neoplastic

\author{
Walled-off necrosis \\ Congenital cyst \\ Retention Cyst \\ Mucinous \\ Mucinous cystic neoplasms \\ Non-mucinous \\ Serous cystic neoplasm \\ Solid pseudopapillary neoplasm \\ Cystic pancreatic neuroendocrine neoplasm \\ Acinar cell cystic neoplasm \\ Ductal adenocarcinoma with cystic degeneration
}

Communicating with main pancreatic duct

Non-neoplastic

Pseudocyst

Walled-off necrosis

Neoplastic
Intraductal papillary mucinous neoplasm non-neoplastic (pseudocyst and WON) and neoplastic (IPMN).

\section{Imaging techniques}

Nowadays, CPLs can be detected with most imaging techniques (ultrasound-US, multidetector computed tomography-MDCT, magnetic resonance imaging with magnetic resonance cholangiopancreatographyMRI with MRCP), but for a correct characterization MDCT and MRI are needed. Endoscopic ultrasoundEUS - is another useful imaging technique to correctly characterize CPLs, but its use is mostly managed by gastroenterologists, and thus, its description is beyond the scope of this paper.

MRI with MRCP, thanks to its high contrast resolution and high sensitivity to static fluids on $\mathrm{T} 2 \mathrm{w}$ sequences, is the best imaging technique to assess communication with MPD (which is the key factor to characterize a CPL); on the other hand, MDCT is the best imaging technique to demonstrate the presence, the intra-lesional localization and the size of eventual calcifications, helpful findings in the differential diagnosis; moreover, in elderly and uncooperative patients MDCT with curved multiplanar reconstruction (MPR) post-processing is a valid technique to assess communication with MPD. Both MRI with MRCP and MDCT have high diagnostic performance in differentiating benign from malignant CPLs, with an accuracy ranging from 73 to $81 \%$ for MRI and $75 \%$ to $78 \%$ for MDCT, respectively [13-16].

\section{CPLs non-communicating with MPD: non-neoplastic Pseudocyst}

Pancreatic pseudocyst is a pancreatic and/or peripancreatic fluid collection with well-defined walls containing pancreatic juice or amylase-rich fluid and essentially no solid material. It is considered a delayed (usually $>4$ weeks) complication of interstitial edematous pancreatitis $[11,17]$.

At MRI, pseudocyst is markedly hyperintense on T2w and hypointense on T1w (Fig. 1), very similar to other pancreatic cysts so to make confident diagnosis of pseudocyst, and it is necessary to have correlation with clinical history of acute pancreatitis [18]. Cui et al. in a multicenter study demonstrated that more than $80 \%$ of pseudocysts disappeared or decreased in size during follow-up (Fig. 1d) [19].

However, acute pancreatitis can be caused by cystic tumors (IPMN), or patients with acute pancreatitis may have cystic tumors, so a previous acute pancreatitis cannot completely exclude the presence of a true cystic tumor.

\section{WON}

WON is an encapsulated collection of pancreatic and/or peripancreatic necrosis surrounded by enhancing walls of reactive tissue that occurs $\geq 4$ weeks after onset of necrotizing pancreatitis as result of the organization of an acute necrotic collection [17]. WON contains necrotic fat and/or pancreatic tissue which manifest at MRI as 

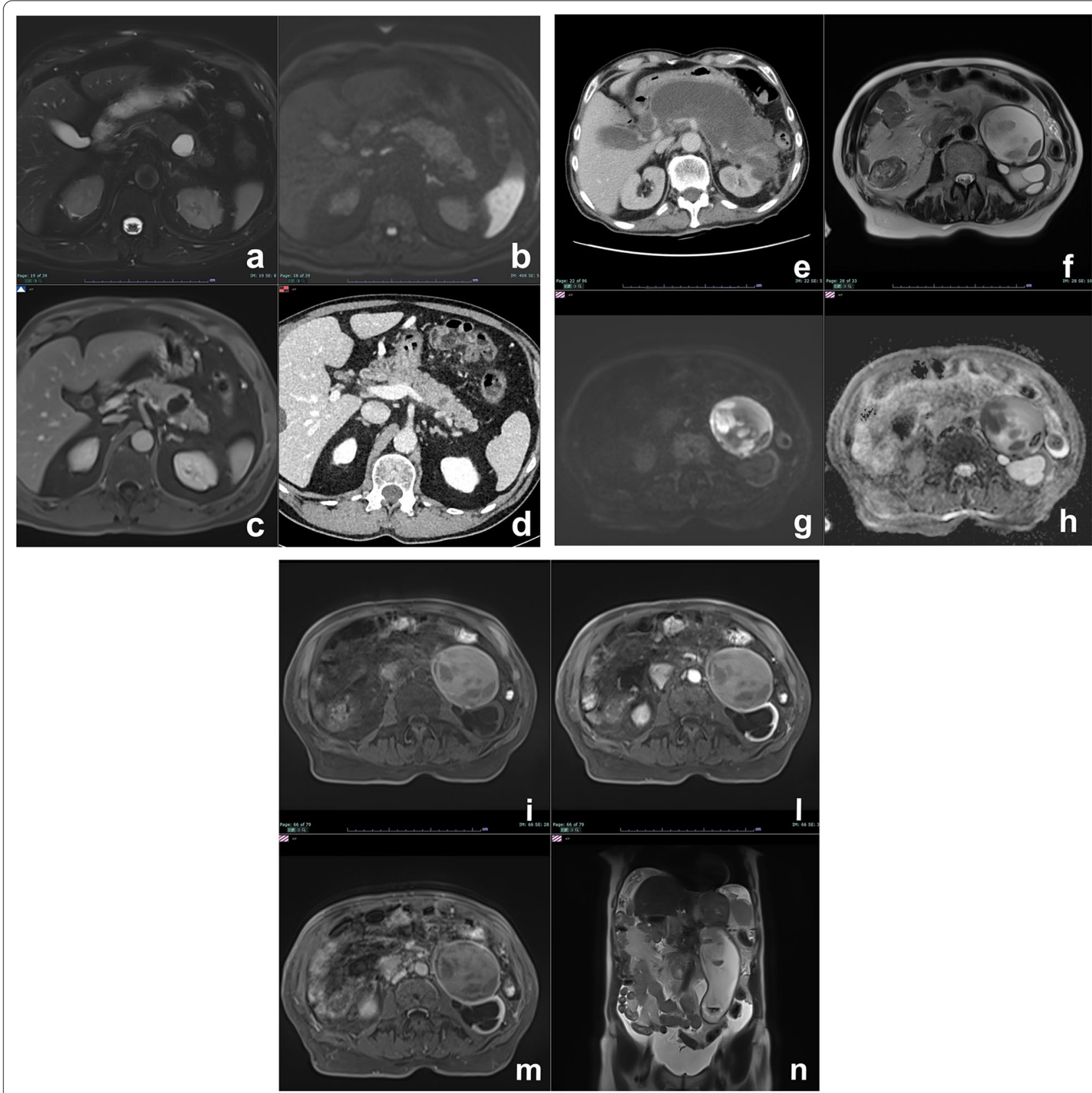

Fig. 1 Cystic complications of acute pancreatitis. a-d Pseudocyst. Patient with a previous interstitial edematous pancreatitis. At follow-up after 1 year, a cystic lesion with no septa or debris is appreciable in the tail of the pancreas, hyperintense on T2w (a), without high signal intensity on high $b$ value DWI (b) and no enhancement after contrast media administration (c). At further follow-up after 6 months, the lesion is decreased in size (d). e-n: WON. A large necrotic collection is appreciable in a patient with necrotizing acute pancreatitis (e). After 1 year, a large collection with debris is still appreciable in the tail of the pancreas, clearly visible on T2w as cystic lesion with low signal intensity foci (f). Debris show inhomogeneous signal intensity on DWI (g), ADC map (h) and T1 unenhanced (i), but do not show any enhancement after contrast media administration (I, $\mathbf{m})$. On T2 HASTE coronal view, the caudal extension of the collection is appreciable (n)

intracystic non-liquefied debris, an highly specific MR finding for the diagnosis of WON (Fig. 1e-n) [11, 20].

\section{Congenital cyst}

Congenital cyst or "true cyst" is an extremely rare cystic lesion lined by a single layer of cuboidal epithelium; it mostly occurs in children or in patients with polycystic disorders (e.g., autosomal dominant polycystic kidney disease) and is usually mistaken or finally diagnosticated by exclusion [21]. 


\section{Retention cyst}

Retention cyst is a cystic dilatation of a pancreatic duct, usually caused by calculi, mucin, chronic pancreatitis or pancreatic cancer.

Retention cyst has mucinous mucosal lining and has imaging features similar to those of small MCN or of BDIPMN [22]. As it can be the first sign of a pancreatic cancer, even if small, it cannot be underestimated and must undergo follow-up (Fig. 2), particularly in young or middle-aged patients [23].
As retention cyst has no characteristic imaging features, it must be considered an indeterminate cyst whose management is different according to different guidelines, based on only the size [9] or balanced on the size and the age of the patient [24]. As a role of thumb, in very small lesions $(<5 \mathrm{~mm})$ a stability after 2-year follow-up is sufficient to stop surveillance [24]; in larger cysts in young ( $<65$ yo) and fit patients, follow-up by imaging should last at least 9-10 years if the lesion is stable [24],

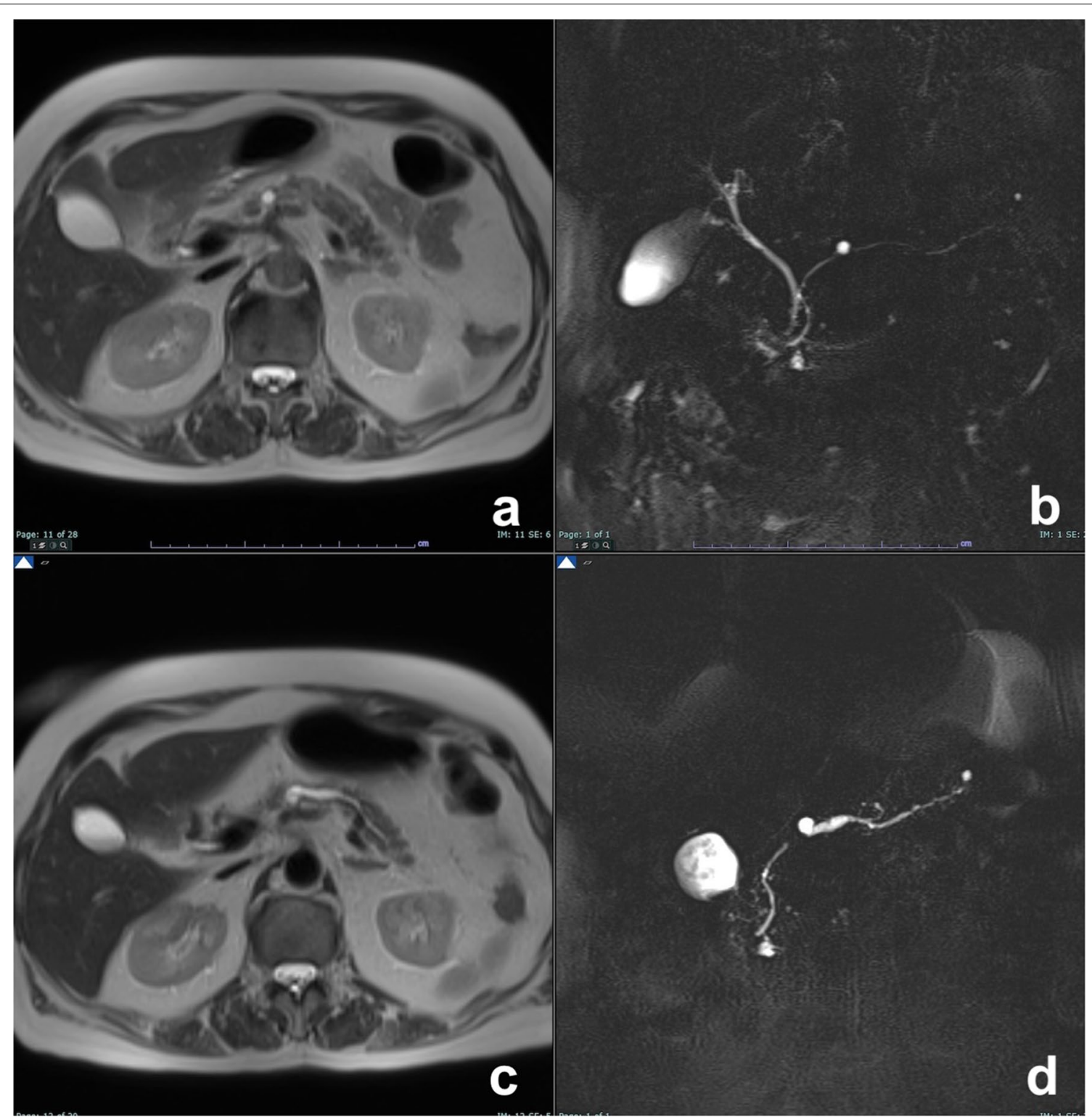

Fig. 2 Retention cyst. A small cyst is observed on T2w (a) and MRCP (b), without evidence of a mass. On a follow-up study two years later, a focal obstruction of the main pancreatic duct appeared with chronic obstructive pancreatitis features upstream (c, d), all findings of high suspicion of malignancy (pancreatic carcinoma on histology). (From: Morana G, Faccinetto A, Venturini S: Pancreas. In: Vanzulli A, Colagrande S, Grazioli L, Morana $\mathrm{G}$ (eds), MRI of the abdomen—technique and imaging findings. Poletto, Milan, 2021) 
while for other guidelines there are not clear indications on the length of follow-up [9].

\section{CPL non-communicating with MPD: neoplastic}

The most common cystic pancreatic neoplasms noncommunicating with MPD are SCN, MCN, SPN, CPNET and $\mathrm{ACCN}$, and these entities can be distinguished on the basis of their morphological features, location and demographic data.

\section{Serous cystic neoplasm (SCN)}

$\mathrm{SCN}$ is a benign lesions, and only very few cases of malignant degeneration have been published [25]. It accounts for $10-15 \%$ of CPLs and for around $1-2 \%$ of all pancreatic neoplasms [26, 27].

It is composed by multiple cysts formed by glycogenrich epithelial cells and mainly involves the head of the pancreas, although it can be located in any part of the gland [28].

It primarily affects females in their $50 \mathrm{~s}$ [26], but it is often detected later.

On $15-30 \%$ of cases, SCN is associated with von Hippel-Lindau disease (VHL) and in these cases tends to be multifocal and may involve diffusely the pancreatic gland [29] (Fig. 3).

SCN is mostly (nearly 60\%) asymptomatic and only when large can cause signs and symptoms of obstructive chronic pancreatitis such as unspecific abdominal pain, diabetes mellitus and pancreaticobiliary symptoms [26].

At imaging, SCN may manifest with three main patterns, reflecting its morphological appearance: microcystic (or polycystic), honeycomb and macrocystic (or oligocystic) [30].

On a multinational review on 2622 patients with SCA [26], authors observed that the most frequent pattern

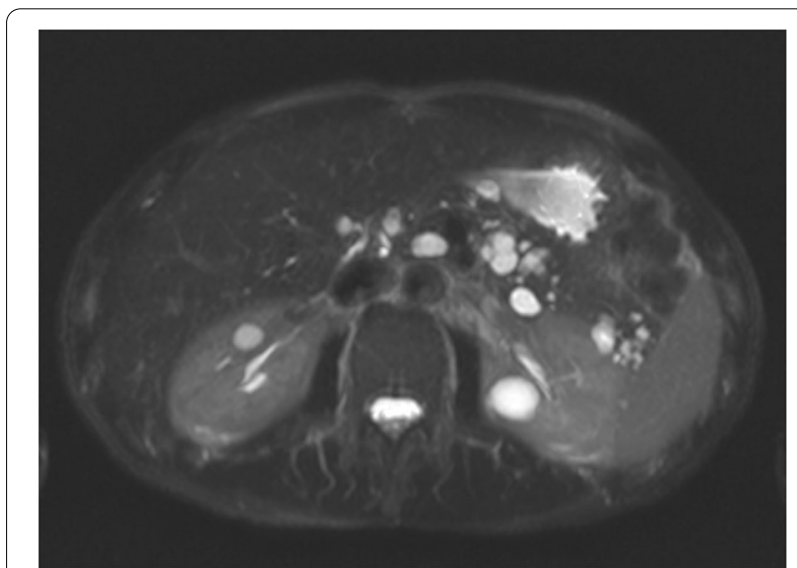

Fig. 3 Von Hippel-Lindau. Several cysts are visible on T2w: multifocal $\mathrm{SCN}$ was microcystic pattern (45\%), followed by macrocystic pattern $(32 \%)$, mixed type $(18 \%)$ and honeycomb pattern (5\%).

The more common microcystic pattern is composed by multiple cysts $<2 \mathrm{~cm}$ separated by fibrous septa that can converge into a central stellate scar that may calcify. In the rarer mixed type, few larger cysts $(>2 \mathrm{~cm})$ are located peripherally. When cysts are numerous and subcentimeters, the lesion assumes an "honeycomb" pattern (Fig. 4) [30].

Some authors observed that central scar had sensitivity $(\mathrm{Se})$, specificity $(\mathrm{Sp})$ and positive predictive value (PPV) of $32.4 \%, 100 \%$ and $100 \%$, respectively, for diagnosis of SCA, while combination of microcystic appearance and lobulated margins had Se, Sp and PPV, respectively, of $68 \%, 100 \%$ and $100 \%$ [31], suggesting that when present, both central scar and combination of microcystic appearance and lobulated margins are pathognomonic of SCN.

Some other authors observed that on MDCT, also the presence of a central calcification and the "circumvascular sign" (i.e., presence of some abnormal arteries surrounding the lesion on arterial phase) is pathognomonic for SCN (100\% Sp), and however, central calcification has a very low sensitivity (less than $30 \%$ ), while circumvascular sign has a Se of 76.7\% [32].

The macrocystic pattern (Fig. 5) is formed by a small number of cysts $\geq 2 \mathrm{~cm}$ without a central scar, and is often indistinguishable from other CPLs, especially MCN and BD-IPMN. Differential diagnosis is important, since SCN is a benign lesion, while MCN and IPMN have a potentially malignant behavior. In a series of 41 CPLs, significant differences in lesion morphology were found among SCN, MCN and IPMN: Macrocystic SCN has multicystic or lobulated contour with or without septation, MCN has smooth contour with or without septation, and IPMN has either a pleomorphic or a clubbed finger-like cystic shape [33]. Besides, SCN may be distinguished from BD-IPMN because the latter communicates with MPD and the communication is usually visible at MRCP.

Rarely also a pseudosolid variant of SCN has been described. Microscopically, the serous solid adenoma shows architectural and cytological characteristics similar to serous microcystic cystadenoma, where the small size of cystic spaces, made by round or ovoid structure formed of cuboid cells derived from the ductular epithelium, is related to the fact that the solid adenoma has no secretory activity, giving a more compact structure compared to cystic forms. Stromal components of these lesions are characterized by avid contrast enhancement, thus misleading to an incorrect diagnosis of neuroendocrine tumor at MDCT (Fig. 6a); however, 


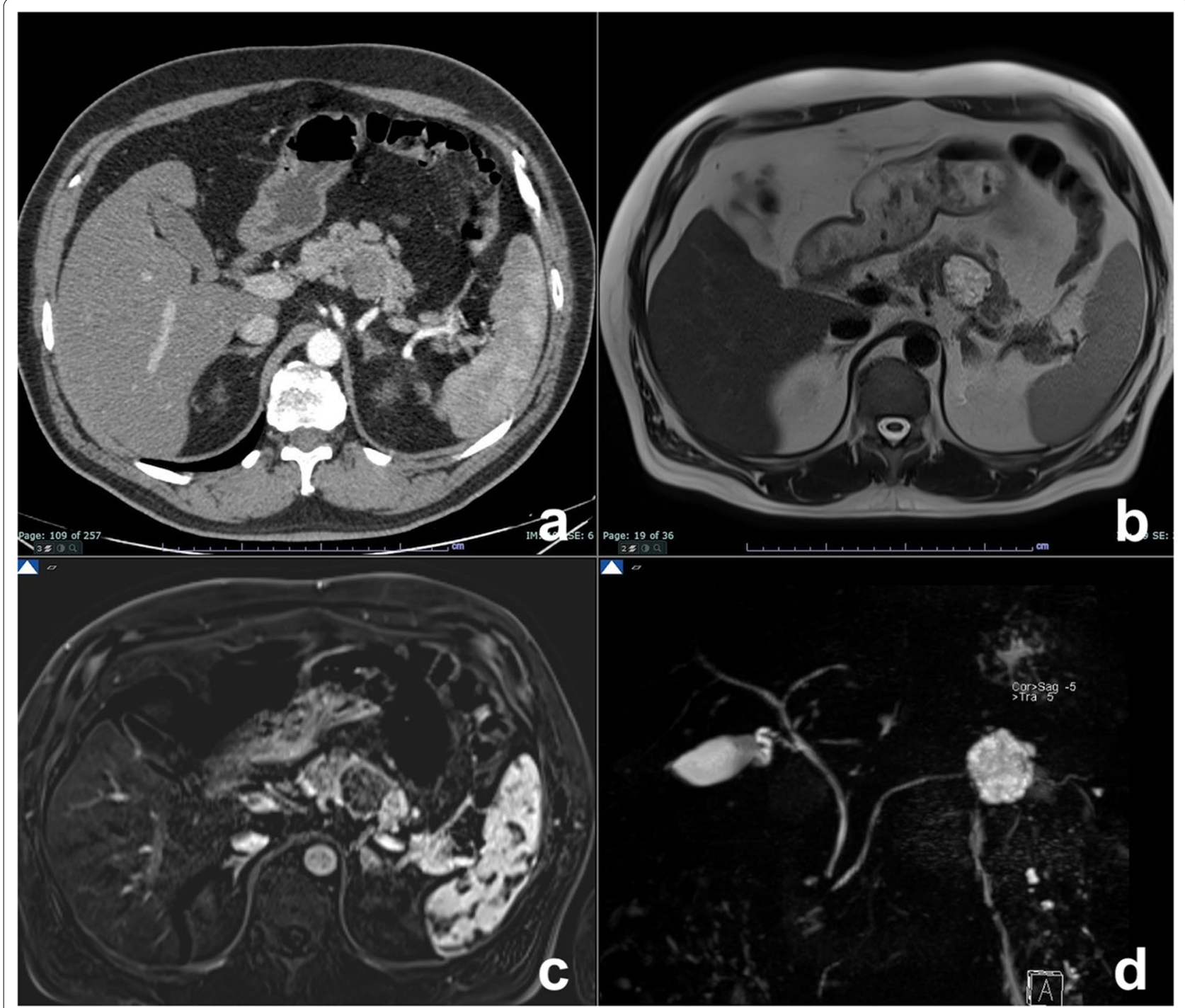

Fig. 4 Serous cystadenoma, "honeycomb" pattern. On contrast enhanced CT (a), a large hypodense lesion with tiny central enhancement can be appreciated in the body-tail of the pancreas. On T2w MRI, the lesion is hyperintense (b). On T1w dynamic imaging, the central enhancement is appreciable, especially with subtraction imaging $(\mathbf{c})$, related to the enhancement of septa and stroma supporting the microcysts. MRCP (d)

MRI, thanks to its high sensitivity to static fluids, can easily characterize this variant [34, 35] (Fig. 6).

On a multinational review on 2622 patients with SCN [26], Jais et al. observed that the SCN-related mortality was almost null, and thus, conservative treatment is suggested for these lesions.

Although recent guidelines do not recommend it [9], follow-up of SCN is suggested by some authors, as size increase is observed in nearly $40 \%$ of patients and it can lead to chronic obstructive pancreatitis (Fig. 7), thus requiring surgery $[6,26,36]$.

\section{Mucinous cystic neoplasm (MCN)}

MCN is a cystic-forming epithelial neoplasm which accounts for $10 \%$ of CPLs and which can be classified into MCN with low-intermediate grade of dysplasia, MCN with high grade of dysplasia and MCN with an associated invasive carcinoma [37].

This lesion is composed by mucin-producing epithelial cells supported by ovarian-type stroma, and thus, it occurs almost exclusively in females (99.7\%), and it is almost always located in the pancreatic body or tail $(94.6 \%)$ [6, 38, 39]. 


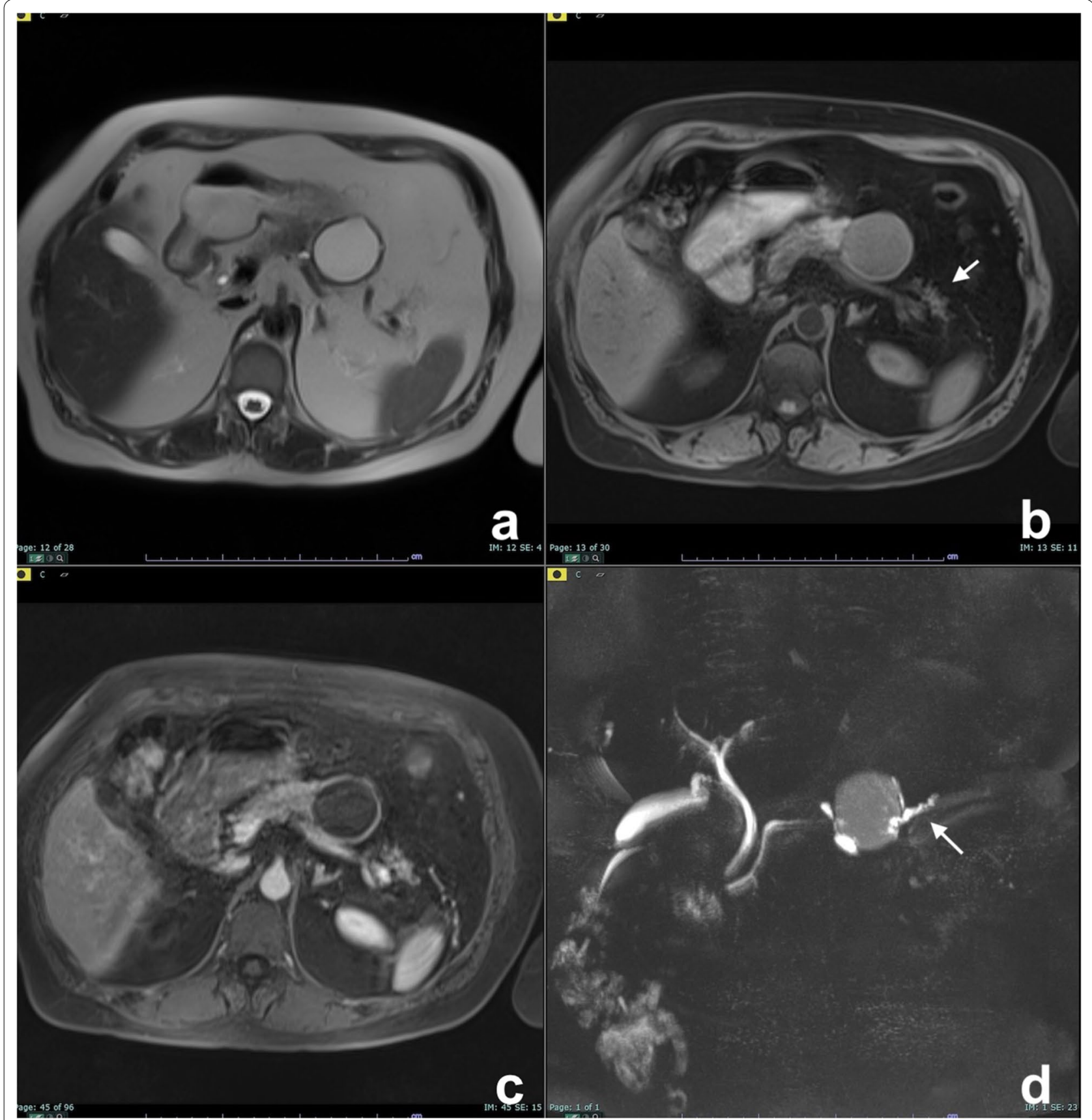

Fig. 5 Serous cystadenoma, macrocystic pattern. Female, 57 yo. A large cist with thick walls is visible in the body of the pancreas with no nodules or enhancing septa both on T2w (a) and T1w before (b) and after contrast media administration (c) with atrophy of the tail (arrow in b). At MRCP (d), a dilatation of the MPD upstream is recognizable (arrow). Due to the high suspicious of a mucinous cystadenoma, the lesion was resected, and at pathology, a serous cystoadenoma was diagnosed

The peak of incidence of MCN is in the $40 \mathrm{~s}$ [6]; however, it can be diagnosed in a wide range of ages with worse degree of malignancy in advanced ages; this suggests progression from benign to malignant lesions, as confirmed by the concomitant presence of different degrees of differentiation, from benign to overtly malignant, in the same lesion $[6,40]$.
As for SCN, MCN may manifest with unspecific symptoms such as abdominal pain and discomfort, seldom referred to the pancreatic region. Only advanced malignant lesions may manifest with more evident clinical signs such as dyspepsia, pain, weight loss and jaundice [37]. 


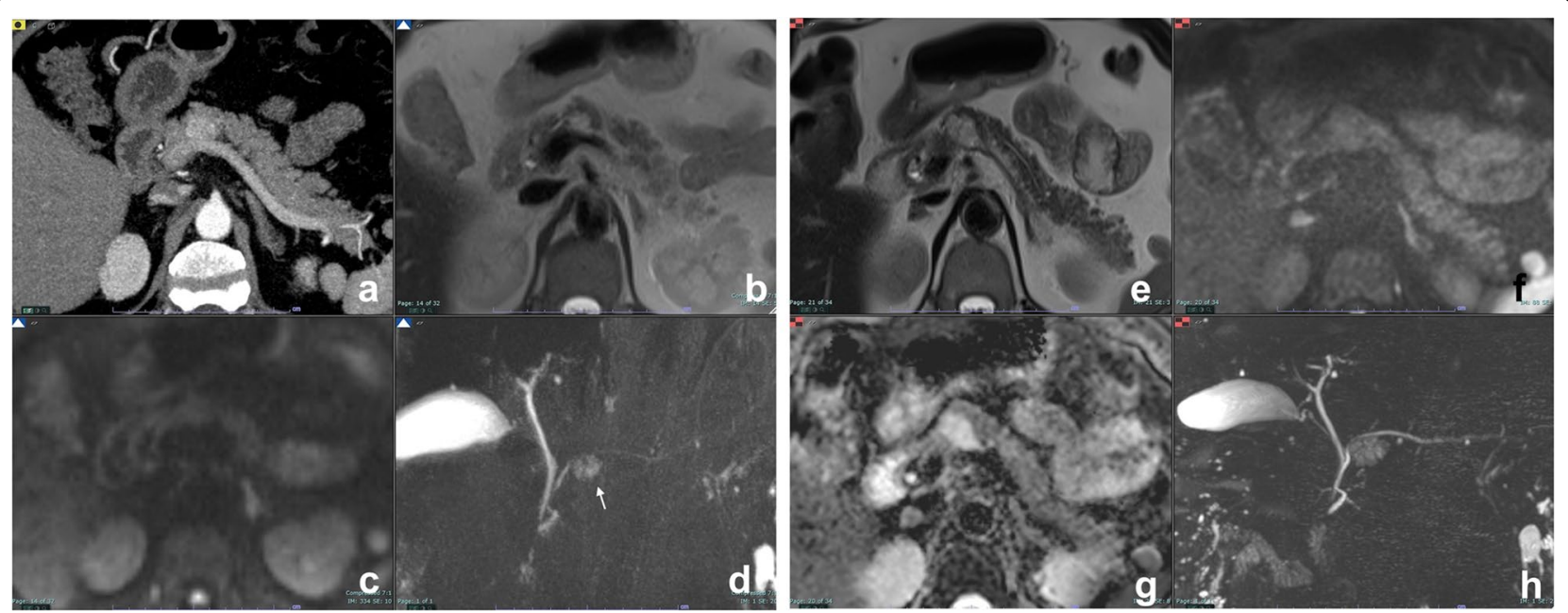

Fig. 6 Serous cystadenoma, pseudosolid pattern. A small hypervascular lesion in the body of the pancreas is visible at contrast-enhanced CT, arterial phase (a). On T2w, the lesion is hyperintense (b), with no high signal intensity at DWI b 800 (c). At MRCP (d), the cystic components are well recognizable (arrow) and MPD is normal. e- $\mathbf{h}$ Follow-up at 5 years. A slight increase in size can be appreciable with no others significant changes. At ADC map (g), the lesion is clearly hyperintense due to cystic content

At imaging, MCN appears as a round cystic masse with sharp margins whose content may have high signal intensity on T1w images due to the presence of mucin and hemorrhage (Figs. 8, 9) [37].

Two main patterns of MCN are described: macrocystic multilocular and macrocystic unilocular [37]. Macrocystic multilocular pattern (Fig. 8) is the most common and typical aspect of $\mathrm{MCN}$, while $\mathrm{MCN}$ with macrocystic unilocular pattern may be confused with other CPLs [37], such as macrocystic SCA (Fig. 5) and pseudocyst (Fig. 1) [41]; as a matter of fact, the diagnosis of a single unilocular cystic pancreatic lesion needs correlation with clinical and with epidemiological data (previous history of acute or chronic pancreatitis, age, gender, site of the lesion) and sometimes remains undetermined, thus requiring more invasive diagnostic tools, such as endoscopic ultrasonography-guided aspiration and biopsy [42].

Radiological signs statistically associated with malignancy are: papillary vegetations, nodules, septa and wall thickness $>3 \mathrm{~mm}$, size $>7 \mathrm{~cm}$, number of loculations $>4$, hyperintensity of cystic content on T1w images, compression and/or infiltration of adjacent vessels or organs and metastases $[37,43]$. The best cutoff value to identify malignant degeneration is the presence of three of these features, with an accuracy of $91 \%$ [43].

In fit patients, resection is recommended also in $\mathrm{MCN}$ without radiological features of malignant degeneration, due to its high malignant potential. However, in elderly patients with comorbidities follow-up may be an option if lesion is smaller than $4 \mathrm{~cm}$ and if mural nodules are absent [44].
Surveillance is recommended with MRI, EUS or a combination of both every 6 months for the first year and then annually if no changes are observed [9].

\section{Solid pseudopapillary neoplasm (SPN)}

SPN is a rare pancreatic cystic tumor (accounting for $1-2 \%$ of all exocrine pancreatic tumors) with a low malignant potential (more than $80 \%$ SPN are benign) and favorable prognosis [45].

This tumor is solid and appears as a round, well-encapsulated mass with a variable amount of necrosis and hemorrhage, responsible of its frequent cystic appearance [45], and sometimes (29\%) it may contain calcifications [46]. It usually affects young women in their $30 \mathrm{~s}$ and has no site predilection, even if they are more frequently located in the tail [45].

This lesion is usually discovered incidentally, as most patients with SPN are asymptomatic, but sometimes it may manifest with unspecific symptoms such as abdominal pain together with palpable mass [45, 47].

At MRI, SPN appears as a well-defined round mass, with heterogeneous signal intensity on both $\mathrm{T} 1$ and $\mathrm{T} 2 \mathrm{w}$ sequences due to necrosis and hemorrhage. Solid components are typically located in the periphery of the lesion, appear well vascularized and enhance later than pancreatic parenchyma (Fig. 10) [46]. When present, acute and subacute hemorrhage is easily recognizable with high signal intensity in T1w sequences and fluid-debris level.

When large cystic changes are present (Fig. 10), SPN appears as a macrocystic uni- or multilocular lesion, 


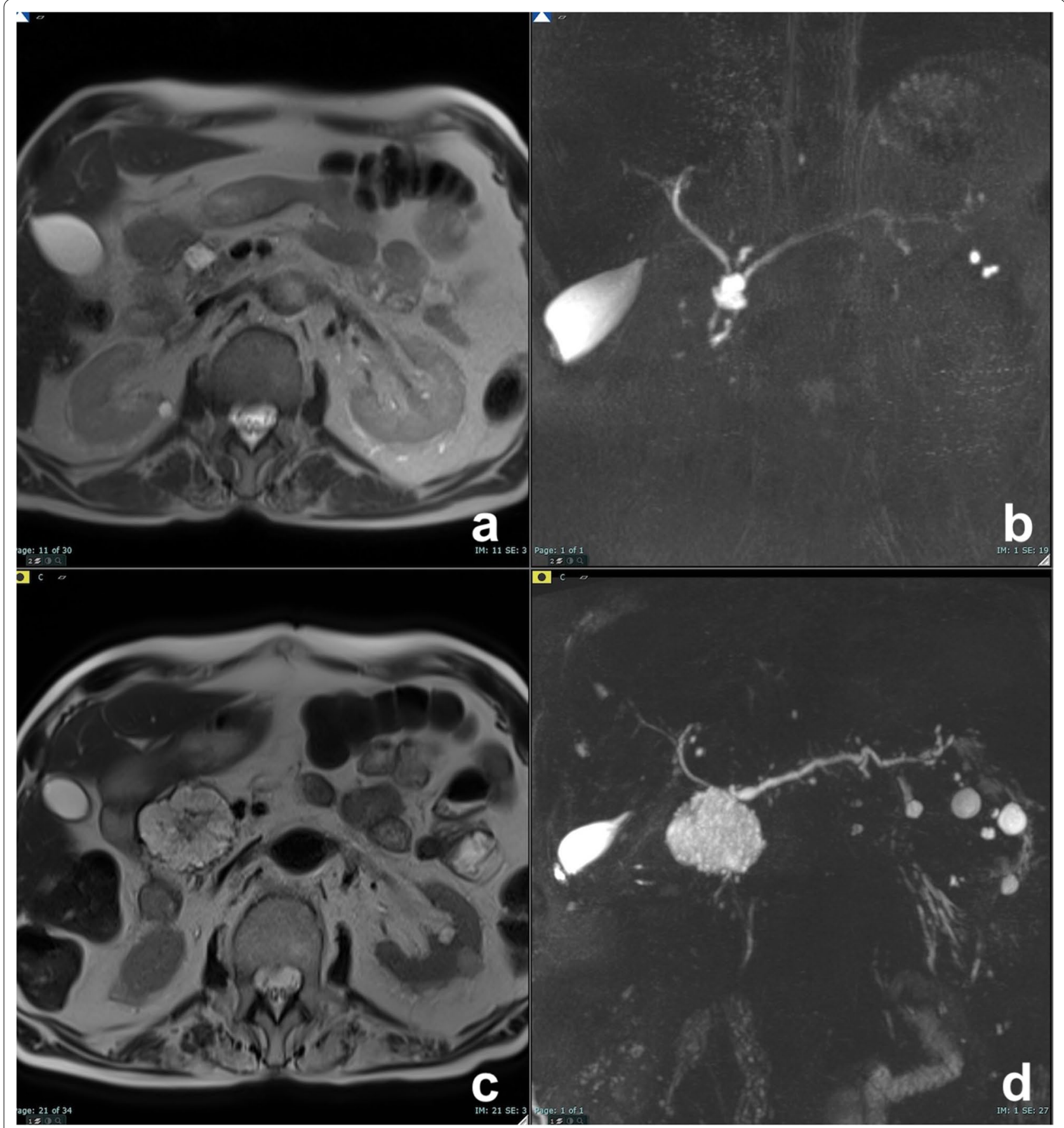

Fig. 7 Microcystic serous cystadenoma with chronic obstructive pancreatitis. On T2w (a), a small lesion not clearly characterizable is appreciated in the neck of the pancreas. At MRCP (b), the wirsung duct is normal. c, d: Follow-up after 12 years: On T2w (c), the lesion is increased in size with a microcystic appearance and a large central scar, typical of a serous cystadenoma. At MRCP (e), a dilatation of wirsung duct with an appearance of chronic obstructive pancreatitis is clearly visible

similar to MCN [48]; in this situation, the younger age of the patient lays for SPN. As it usually exhibits benign behavior, it can be treated with pancreatic sparing resection [49].

\section{Acinar cell cystic neoplasm (ACCN)}

ACCN is a very uncommon benign cystic lesion characterized by serous content and prominent acinar cell differentiation of the epithelial lining, unrelated to the major ductal system [50]. 

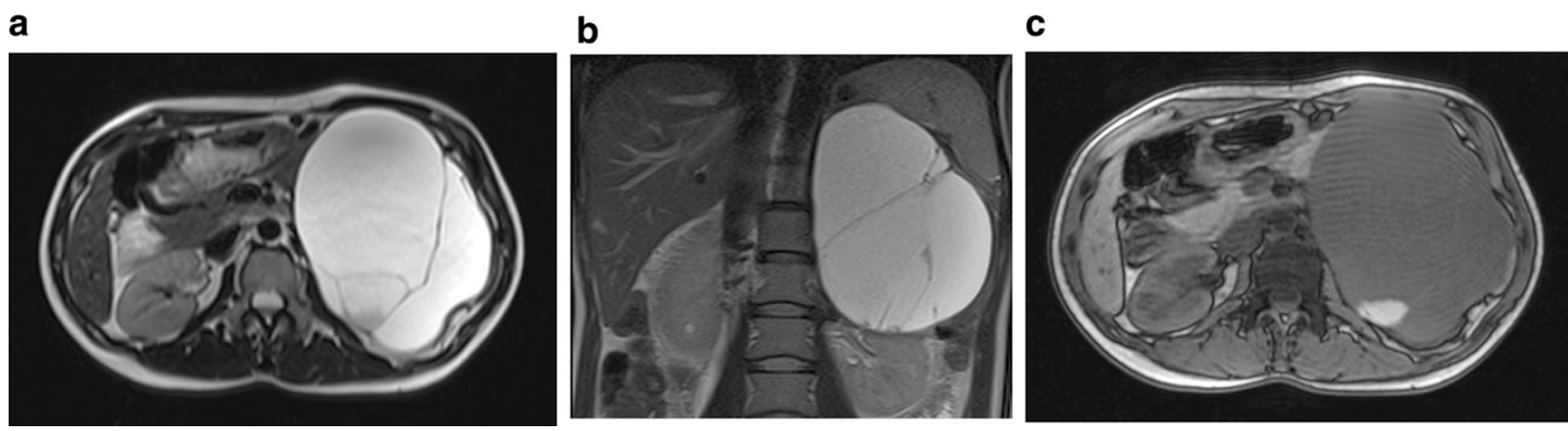

Fig. 8 Mucinous cystadenoma, multilocular pattern. On T2w axial sequence (a), a large multilocular cyst is appreciable in the tail of the pancreas. The multilocular pattern is better appreciable on coronal T2w sequence (b). Administration of paramagnetic contrast media was avoided as patient was pregnant. On axial T1w scan (c), an hyperintense deposit is appreciable in the lower part of the cyst, corresponding to mucin deposit

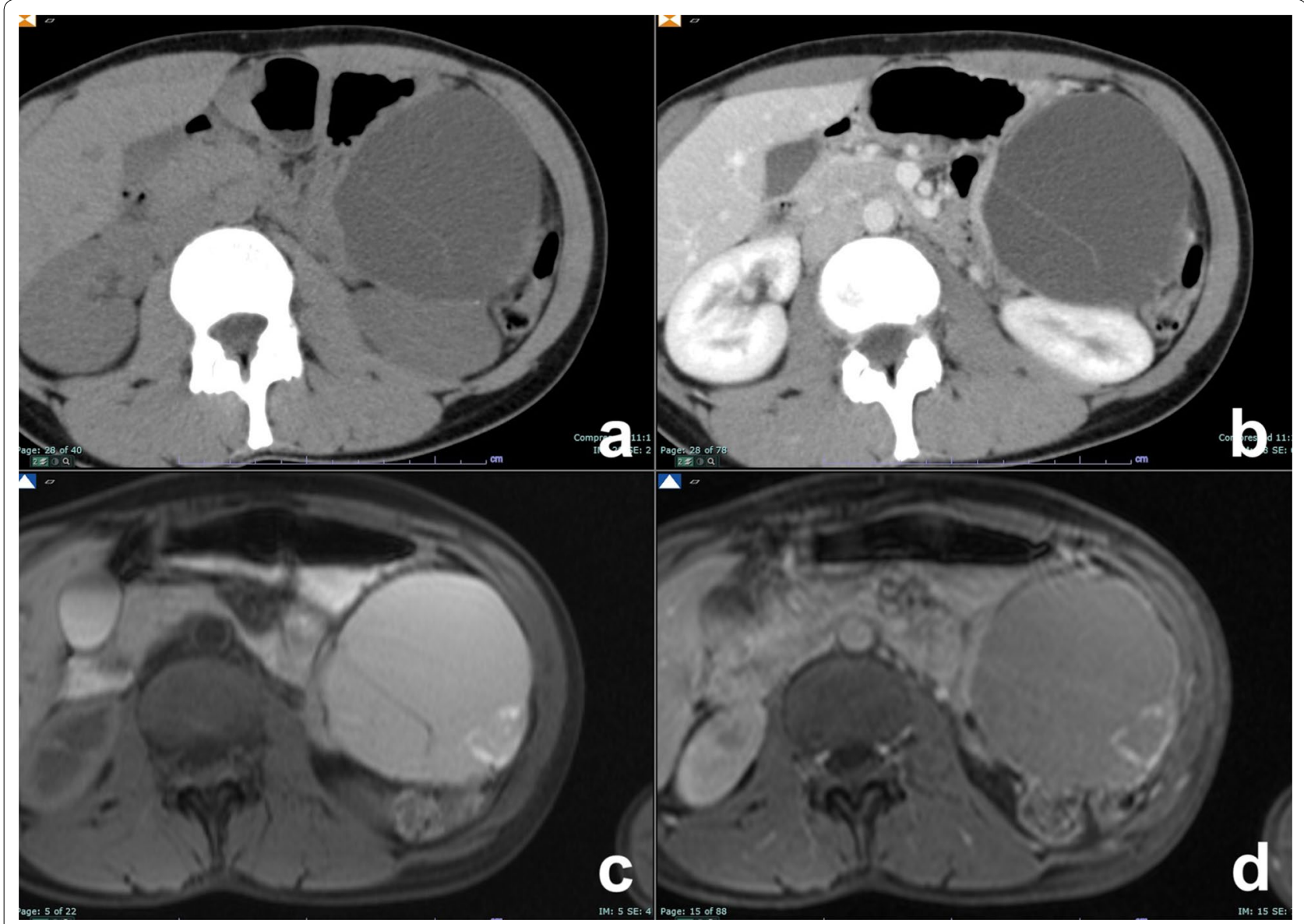

Fig. 9 Mucinous cystadenoma, multilocular pattern. At unenhanced CT (a), a large cyst with a tiny septum is visible in the tail of the pancreas, which shows a slight enhancement after contrast media administration (b). On T1w fat-saturated GRE image (c), some hyperintense foci are visible in the inferolateral aspect of the cyst, which do not show significant enhancement after injection of paramagnetic contrast media (d), related to mucin content

Its exact nature is still unclear: Initially described as a non-neoplastic entity such as developmental anomaly or post-obstructive glandular dilatation and referred as "acinar cystic transformation" (ACT), subsequent studies considered it a neoplastic lesion, so further studies are necessary to clearly demonstrate dystrophic versus neoplastic origin [51]. 


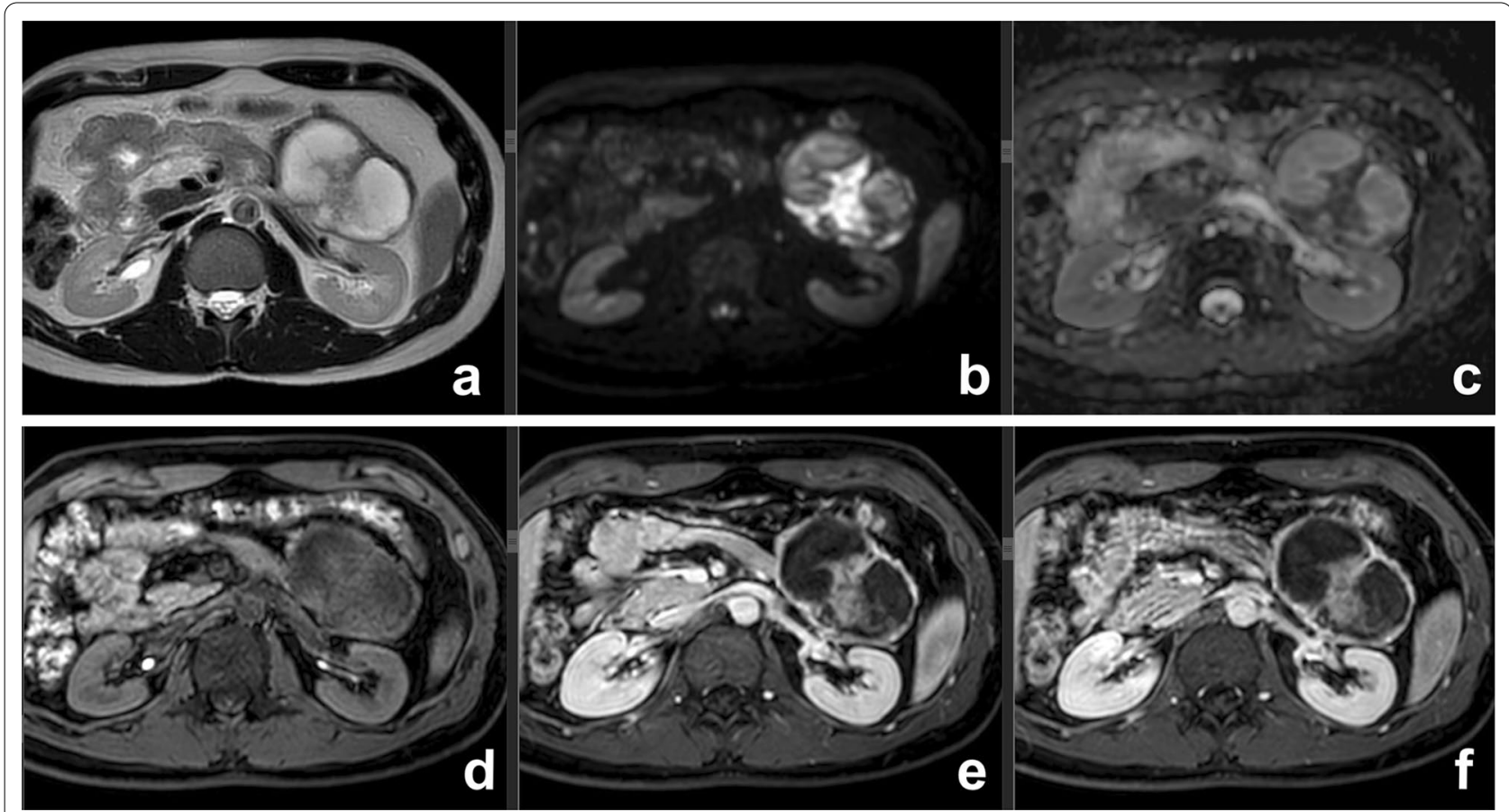

Fig. 10 Solid pseudopapillary neoplasm. On T2w (a), a large cystic lesion with solid components is appreciable in the tail of the pancreas. The solid component shows high signal on DWI b 800 (b) and restricted diffusion at ADC map (c) and contrast enhancement on T1w after paramagnetic contrast media administration $(\mathbf{d}-\mathbf{f})$

It can manifest as unilocular and, more frequently, multilocular cyst which typically lacks solid areas of papillary projections and which can either be confined to an anatomic region or diffusely involve the entire gland [50-52].

It can occur in a wide range of ages with a striking female predominance and causes clinical symptoms in half cases, in particular abdominal pain.

Preoperative diagnosis is usually based on a combination of clinical features and radiological findings, but is often difficult because of similarity to other cystic pancreatic lesions. In particular, radiological findings of unilocular ACCN overlap with those of MCN and macrocystic $\mathrm{SCN}$, while the main differential diagnosis of multilocular ACCN is IPMN. Delavaud et al. demonstrated that four radiological findings are significantly associated with ACCN (five or more cysts, clustered peripheral small cysts, presence of cyst calcifications and absence of communication with the main pancreatic duct) (Fig. 11) and that the presence of at least two or three of these imaging criteria had a strong diagnostic value for ACCN with a Se of $100 \%$ and $80 \%$ and a Sp of $85 \%$ and $100 \%$, respectively [53].

As there have been no reports of malignant transformation, it is crucial to correctly characterize ACCN since it requires conservative treatment (even if pathological confirmation is still needed) [50-52].
When diagnosis of ACCN is suspected, patients should undergo surgical biopsy under laparoscopy with frozen section examination (the diagnosis of ACCN with US-guided fine needle aspiration is very difficult, since ACCN lining cells are a true mimic of normal acinar cells) and once the diagnosis is confirmed, patients must be followed up, because the exact nature of ACCN (dystrophic or neoplastic) is not clearly defined yet [53].

\section{Cystic pancreatic neuroendocrine tumor (CPNET)}

Cystic pancreatic neuroendocrine tumor (CPNET) is a distinctive subgroup of pancreatic neuroendocrine tumors (PNETs) with unique clinical, radiological and pathological features [54-56].

Its etiology is not clear yet. On gross pathology, it usually appears as well-demarcated lesions with centrally or eccentrically located single thin-walled cystic area containing clear fluid which does not communicate with pancreatic ducts and which is separated by a thin fibrous band from neoplastic cells.

Different authors demonstrated that compared with solid PNET, CPNET is more likely non-functioning, solitary and more likely associated with MEN1 syndrome. It is less likely to demonstrate tumor necrosis, perineurial invasion, vascular invasion, regional lymph node metastases and synchronous distant metastases 


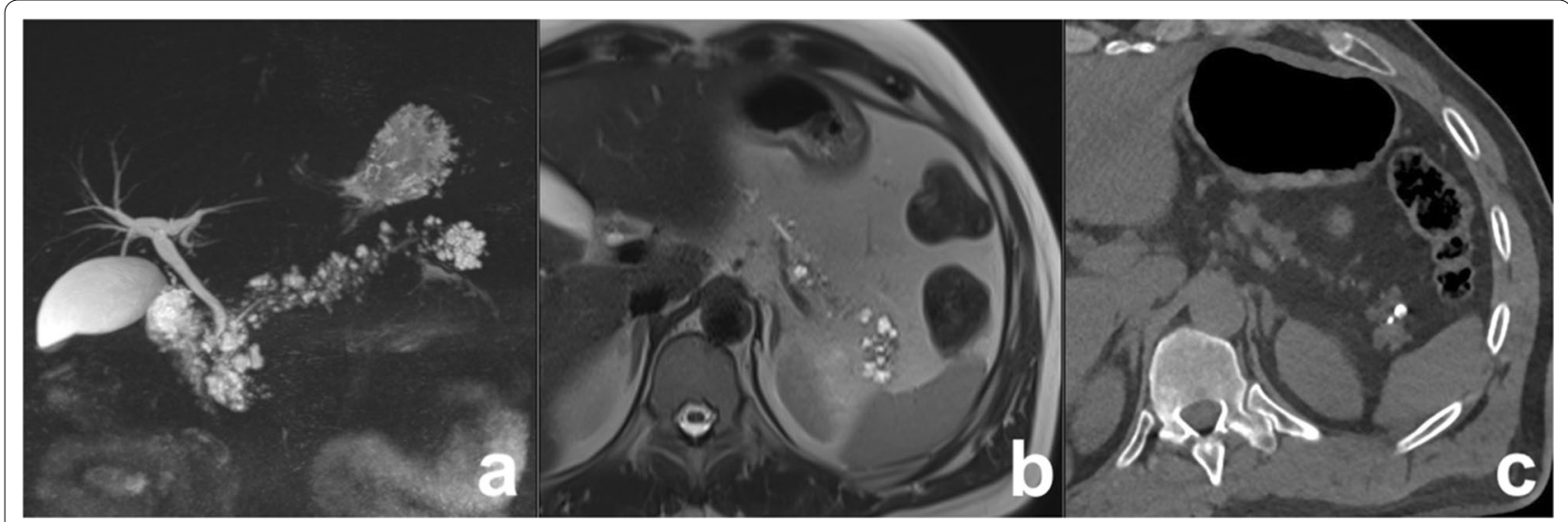

Fig. 11 Acinar cell cystadenoma. At MRCP (a), there are several small cysts thought the pancreas. On T2w (b), some small hypointense foci are appreciable in the tail of the pancreas which at CT show up to be calcifications (c). At biopsy, an acinar cell cystoadenoma was found

and present with a lower pathological stage at diagnosis, with low Ki-67 index and mitotic count [54-56]. Moreover, patients with resected CPNET have a better 5-10-year disease-free survival compared with solid PNET [56].

It occurs over a wide range of ages, more often in adults (mean age: 53 years) and in men, and it accounts for $3.6-36.1 \%$ of resected PNETs [56].

At CT or MRI, CPNET appears as non-communicating cystic lesion surrounded by a rim of well-vascularized tissue that enhances in the arterial phase [57] (Fig. 12). The main differential diagnosis is with $\mathrm{MCN}$ and BD-IPMN [55].

Because of its indolent behavior, more conservative surgical procedures are proposed such as tumor enucleation and spleen preserving distal pancreatectomy, and in some selected patients, particularly those with purely cystic tumor $<2 \mathrm{~cm}$, only surveillance is suggested [58-60]. However, preoperative radiological diagnosis of CPNET is difficult with a misdiagnosing rate up to $50 \%$ even in high-volume centers specialized in pancreatic tumors $[55,56]$; anyway, when operators are expert and when sufficient material is present for immunohistochemical staining, preoperative diagnosis can be achieved by EUS-fine needle aspiration with a diagnostic accuracy up to $100 \%[55-57,61]$.

\section{CPLs communicating with MPD: IPMN}

IPMN represents a large and heterogeneous group of epithelial mucin-producing tumors communicating or involving the main pancreatic duct.

It is the most common $\mathrm{CPN}$, accounting for about $70 \%$ of all pancreatic cystic neoplasms, may be multifocal and have a malignant potential following an adenoma-carcinoma sequence. IPMN can display the full spectrum of histologic changes, from hyperplasia, adenoma, borderline tumor to in situ or invasive carcinoma, with a different incidence between main duct IPMN (MD-IPMN) and branch duct IPMN (BD-IPMN) $[62,63]$. Moreover, it is characterized by a unique feature such as increased de novo development of pancreatic ductal adenocarcinoma (PDAC) elsewhere in the pancreas, suggesting the presence of diffuse pathologic changes predisposing to malignant transformation.

According to the cell lineage of the papillary component, four histological subtype of IPMN with different clinical pathological behavior can be distinguished: gastric, intestinal, oncocytic and pancreatobiliary [62, 64].

The gastric subtype, which accounts for the vast majority of BD-IPMNs, is typically of low grade with only a small percentage developing into carcinoma; however, if a carcinoma does develop, it is usually of the tubular type and behaves like a conventional PDAC.

A significant portion of MD-IPMN is of the intestinal type, and large and complex intestinal-type IPMNs can have invasive carcinoma, typically of the colloid type with a relatively indolent behavior.

The oncocytic type tends to be large, has relatively uncommon and limited invasion, seems to have a very good prognosis and tends to recur in the remaining pancreas years after the initial resection.

The pancreatobiliary type is the least common and is regarded as a high-grade version of the gastric type. Invasive carcinoma associated with pancreatobiliary-type IPMN is usually tubular and aggressive.

According to its site, three morphological types of IPMN have been described: the main duct type (MDIPMN), the branch duct type (BD-IPMN) and the mixed type which meets both the criteria for MDIPMN and for BD-IPMN. Frequencies of malignancy 


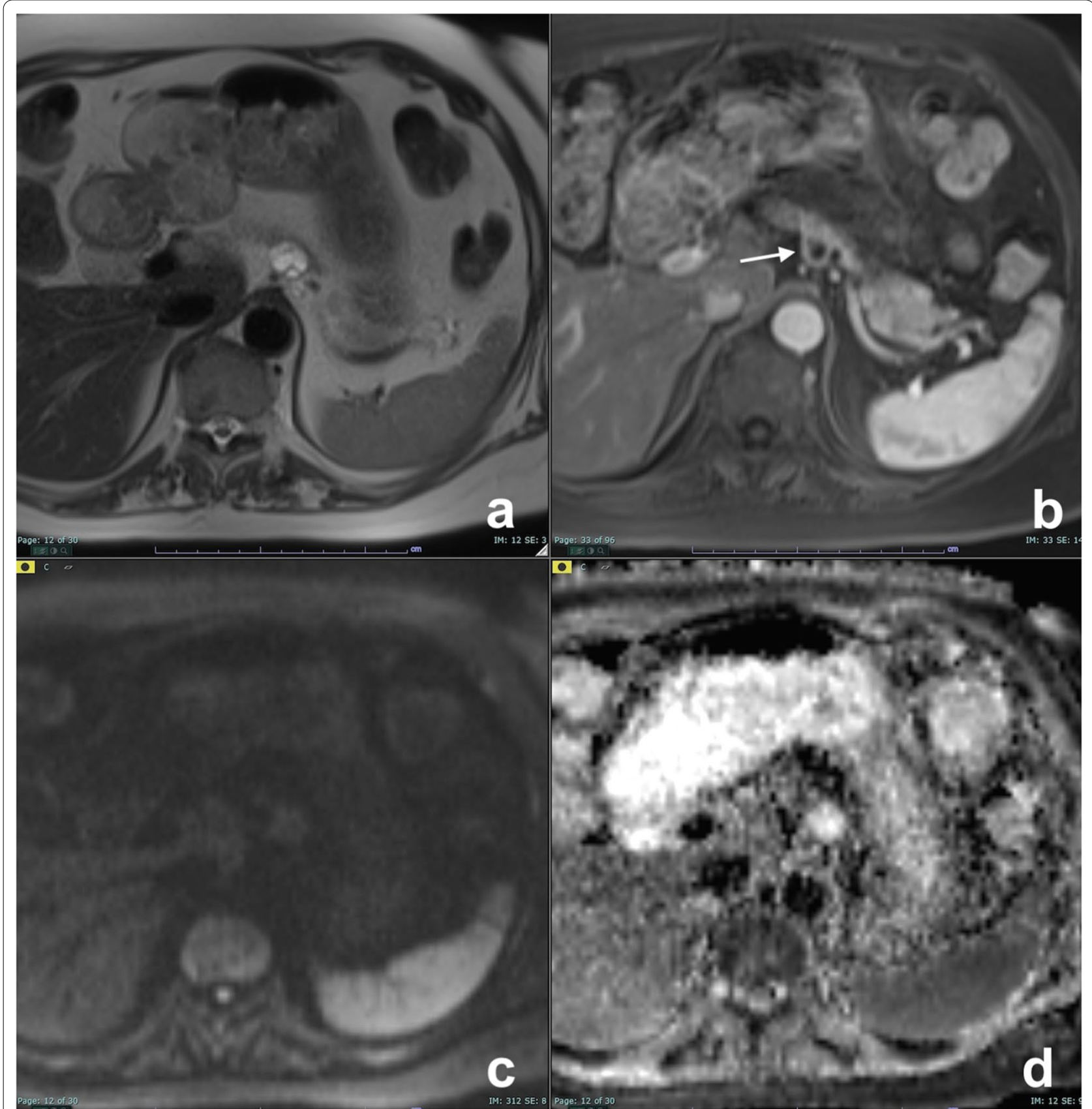

Fig. 12 Cystic PNET. On T2w (a), a small hyperintense lesion with tiny septa is appreciable in the body of the pancreas. AtT1w (b), during injection of paramagnetic contrast media an intense rim enhancement can be appreciable in the arterial phase (arrow). On DWI (c), no restricted diffusion can be appreciated and the lesion appears hyperintense at ADC map (d). After surgical removal, a G1 PNET was diagnosed at pathology

are significantly different according to the morphological types and have been higher for MD-IPMN/ mixed type (mean: 61.6\%) and lower for BD-IPMN (mean: 25.5\%) [62, 64].

IPMN is diagnosed at a mean age of 60 years, and it affects males slightly more frequently than females [62, 63].
IPMN is mostly detected in asymptomatic patients, but sometimes impaired outflow of pancreatic juice induced by hypersecretion of mucin may cause pain, may induce laboratory test abnormalities of pancreatitis and may cause acute pancreatitis itself, and thus, an IPMN may be discovered after an episode of acute pancreatitis. More severe symptoms, such as jaundice, severe abdominal 
pain, anorexia, weight loss and diabetes, are more likely associated with malignant behavior [64].

At imaging, IPMN appears as a cystic dilatation of the involved ductal segment, caused by mucin secretion. MRI is the most sensitive technique to characterize IPMN, and in particular, MRCP is the most important sequence to demonstrate the involvement or the communication of MPD which are the key findings for the appropriate diagnosis of IPMN. Even endoscopic retrograde cholangiopancreatography (ERCP) may fail in demonstrating mucin-filled secondary duct dilatation [62].

MD-IPMN appears as segmental or diffuse dilatation of the MPD (>5 mm) without other causes of obstruction [64]. In diffuse MD-IPMN, differential diagnosis with chronic pancreatitis may be difficult; however, in MDIPMN the dilatation of MPD is more homogeneous with regular margins (Fig. 13). In a comparative study of IPMN and chronic pancreatitis, specific findings for IPMN were duct dilatation without strictures, bulging ampulla, nodule in a duct, grape-like secondary duct dilatation and nodule in a cyst, while specific findings for chronic pancreatitis were ductal dilatation with strictures, the presence of a stone and side branch ectasia with non-cystic appearance (Fig. 14) [65].

Segmental MD-IPMN may be difficult to diagnose because it appears as a non-specific segmental dilatation without an obstruction and without a previous pancreatitis (which can lead to a post-inflammatory stenosis). The affected segment is enlarged, sometimes with dilatation of collateral ducts because of pancreatic juice outflow impairment. In these cases, also upstream dilatation of MPD may be present, thus misleading to an incorrect diagnosis of diffuse MD-IPMN (Fig. 15). In MD-IPMN, parenchyma atrophy is frequently seen (Fig. 15) [66, 67]. If left untreated segmental, MD-IPMN can grow along the main pancreatic duct till a complete involvement (Fig. 16).

Mixed-type IPMN appears as a segmental or diffuse dilatation of MPD and branch ducts along its course (Fig. 16) [68]. However, BD-IPMN may determine dilatation of MPD because of mucin overproduction but not because of MPD involvement, mimicking mixed-type IPMN.

BD-IPMN appears as unifocal of multifocal cystic lesion communicating with MPD. Cysts may be uni(Fig. 17) or multilocular (Fig. 18) with diameter ranging from few $\mathrm{mm}$ to some $\mathrm{cm}$, are often arranged in grapelike clusters and are separated by thin septa that usually enhance after contrast medium administration $[66,67$, 69]. Demonstration of communication with MPD is fundamental to make diagnosis of BD-IPMN (50), and thus, high-quality MRCP is the most sensitive and important sequence of the whole MRI protocol [70].

The main differential diagnosis of unifocal BD-IPMN is pseudocysts, MCN and SCN, since sometimes communication with MPD cannot be correctly assessed $[66,68]$, while the main differential diagnosis of multifocal BDIPMN is with the rare ACCN (Fig. 11) [53].

IPMN has heterogeneous malignant potential, and thus, the International Association of Pancreatology (IAP), with Fukuoka consensus in 2012, proposed twotier criteria to predict malignancy [64].

First tier is represented by "worrisome features," a group of imaging findings suggesting that the lesion is not malignant yet, but could evolve in malignant, and thus requires further work-up by EUS, to better riskstratify the lesion, and strict follow-up (Table 3).

Second tier is represented by "high-risk stigmata," a group of imaging findings suggesting the possibility that

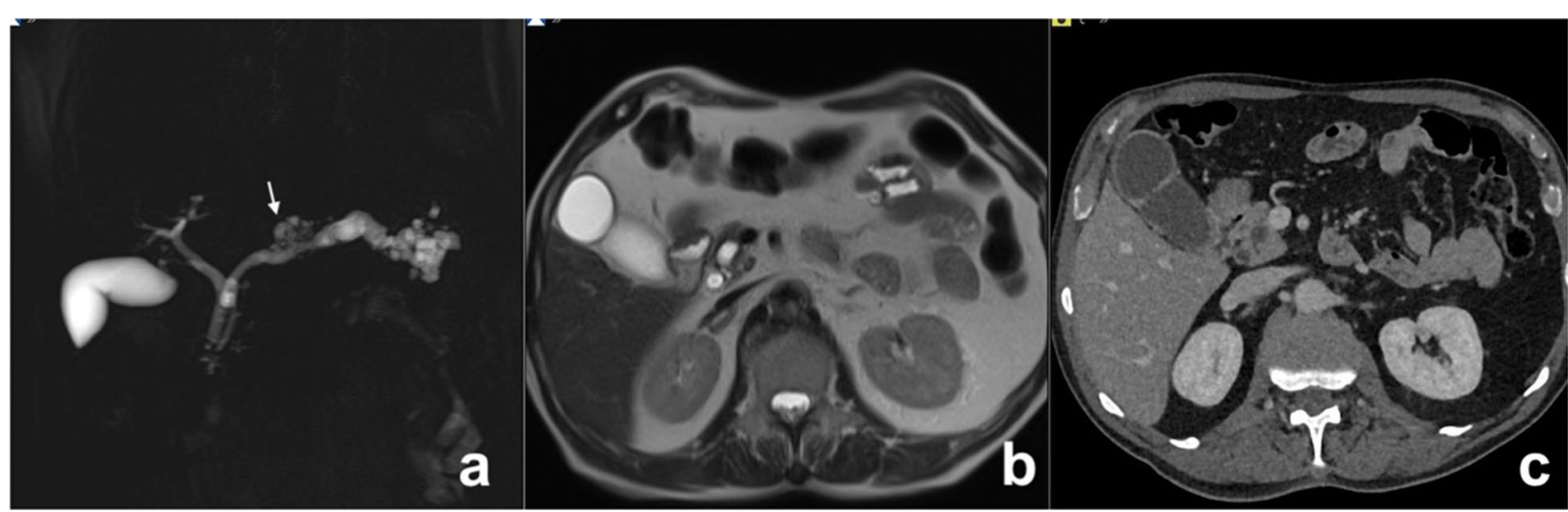

Fig. 13 Diffuse MD-IPMN. At MRCP (a), a diffuse enlargement of the MPD is appreciable through the gland with a grape-like dilatation of branch ducts (arrow) and without significant causes of obstruction nor calcifications both on T2w (b) and CT (c) 


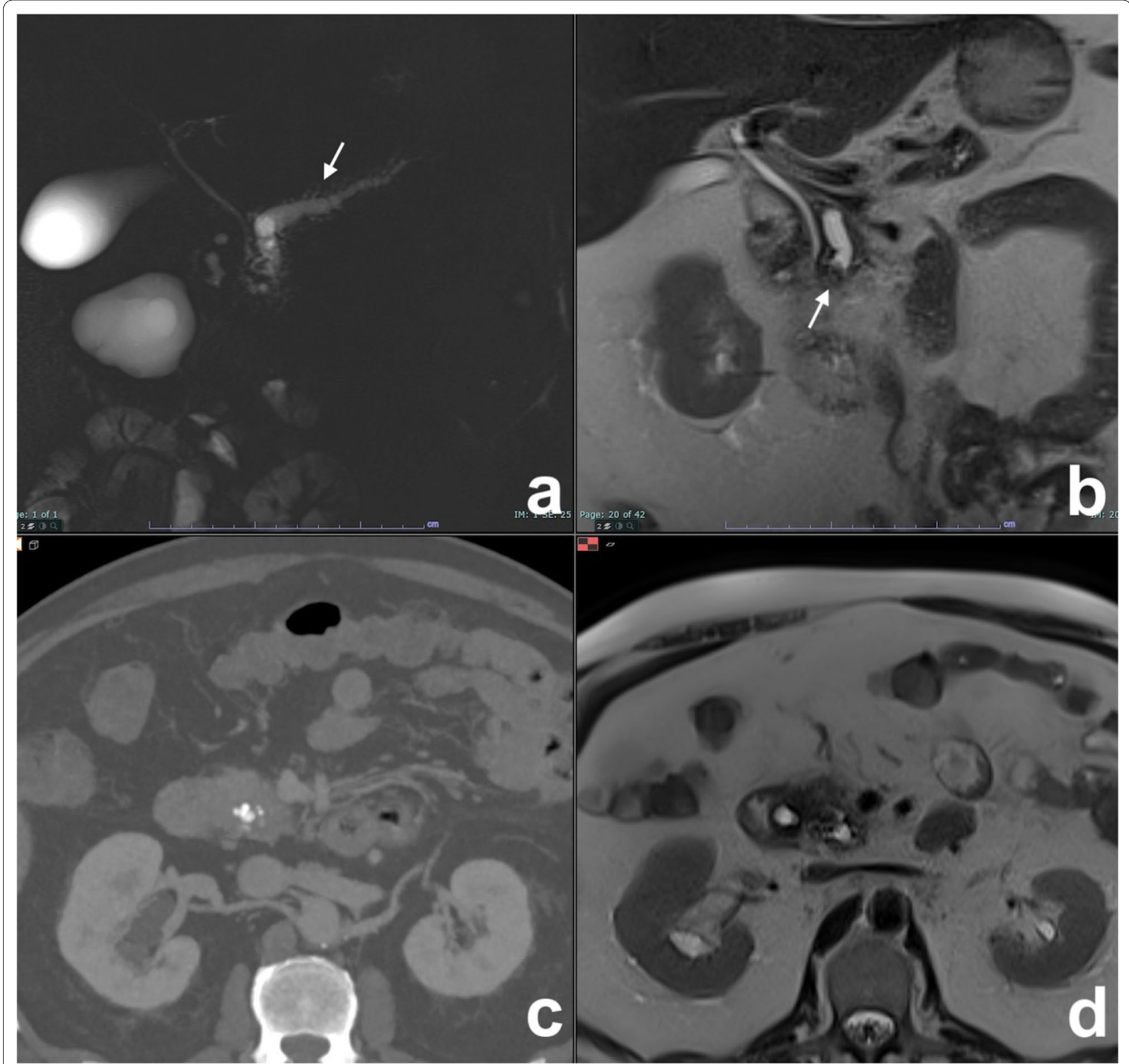

Fig. 14 Chronic obstructive pancreatitis. At $\operatorname{MRCP}(\mathbf{a})$, a diffuse dilatation of the main pancreatic duct is appreciable through the gland with finger-like dilatation of branch ducts (arrow). At T2w, a defect in the prepapillary portion of the wirsung duct is appreciable (arrow). At unenhanced $\mathrm{CT}(\mathbf{c})$, a gross calcification is visible in the head of the pancreas, related to the intraductal calculus, responsible for the dilatation of the wirsung duct upstream, not visible on axial T2w (d)

the lesion is malignant, thus requiring surgical resection if the patient is fit (Table 3).

2012 Fukuoka consensus guidelines had been widely accepted and had high sensitivity to predict invasive carcinoma and high grade dysplasia, but relative low specificity, leading to a high number of unnecessary pancreatic resections, so in 2017 revised Fukuoka consensus has been proposed [71] (Table 3). However, this new consensus needs future cohort studies to demonstrate whether the revised criteria allow to increase specificity without jeopardizing sensitivity [63].

To date, management of IPMN is still controversial; to simplify, according to 2017 Fukuoka revised consensus, patients with IPMN with high-risk stigmata have to undergo resection if fit, patients with IPMN with worrisome features need further workup and patients with IPMN without worrisome features and without high-risk 


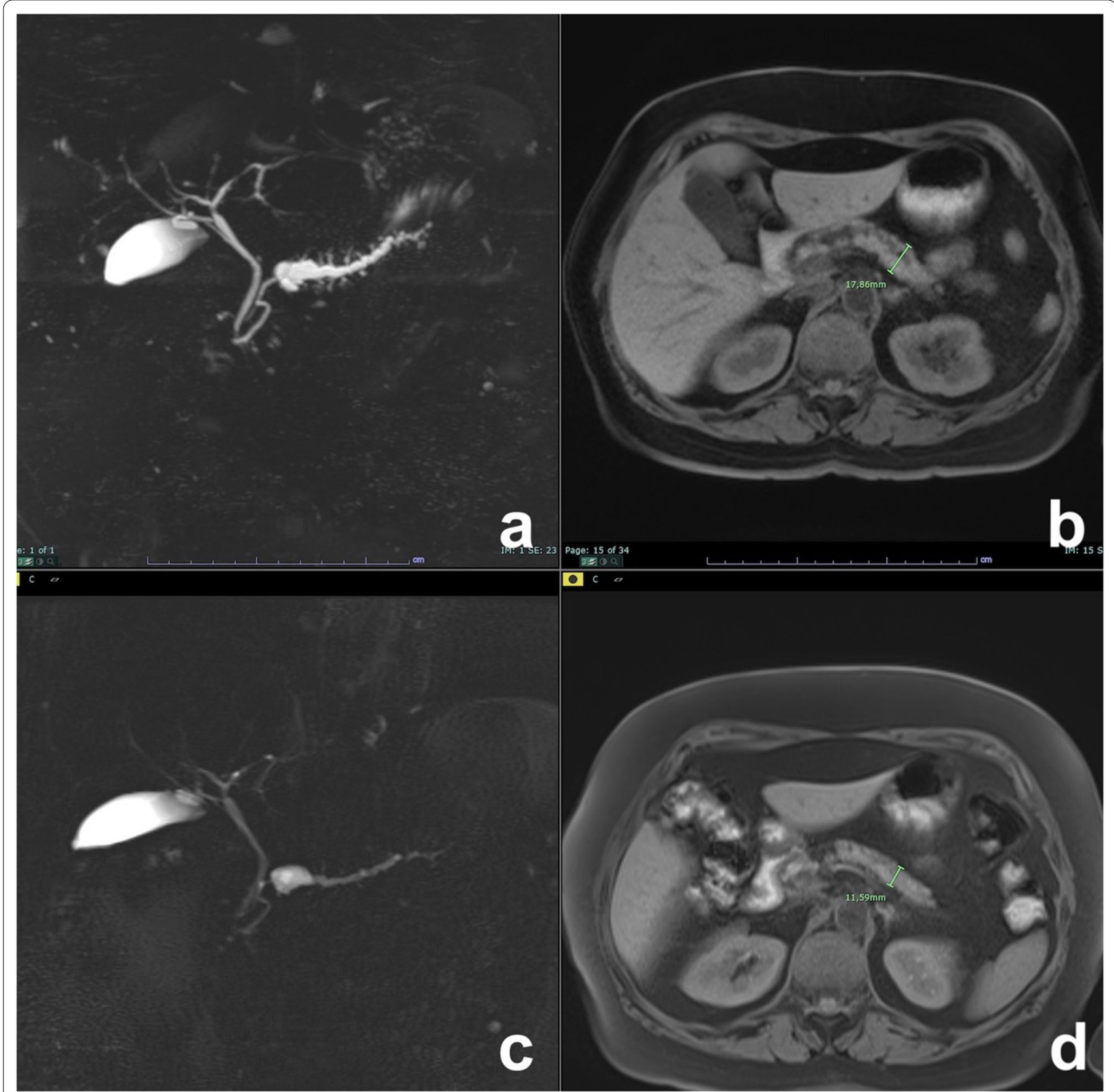

Fig. 15 Segmental MD-IPMN. At MRCP (a), a segmental dilatation of the MPD is appreciable in the body of the pancreas. The MPD upstream shows signs of chronic obstructive pancreatitis (finger-like dilatation of branch ducts), but the thickness of the pancreatic gland is normal (b). After 20 months, the dilatation of the MPD upstream is no longer appreciable (c), due to atrophy of the pancreatic gland (d)

stigmata need follow-up at different intervals depending on the size of the largest cyst (Table 4).

European guidelines distinguish absolute and relative indications for surgery (Table 5). Patients with absolute indications for surgery, patients with one or more relative indications for surgery and without significant comorbidities and patients with comorbidities and with two or more indications for surgery should be operated, while patients with significant comorbidities or short life expectancy and with only one relative indication for surgery should undergo intensive surveillance with clinical evaluation, serum CA 19.9, MRI and/or EUS every six months. Patients without indications for surgery should be followed up with clinical evaluation, serum CA 19.9, MRI and/or EUS every six months for the first year from diagnosis and then yearly [9] 


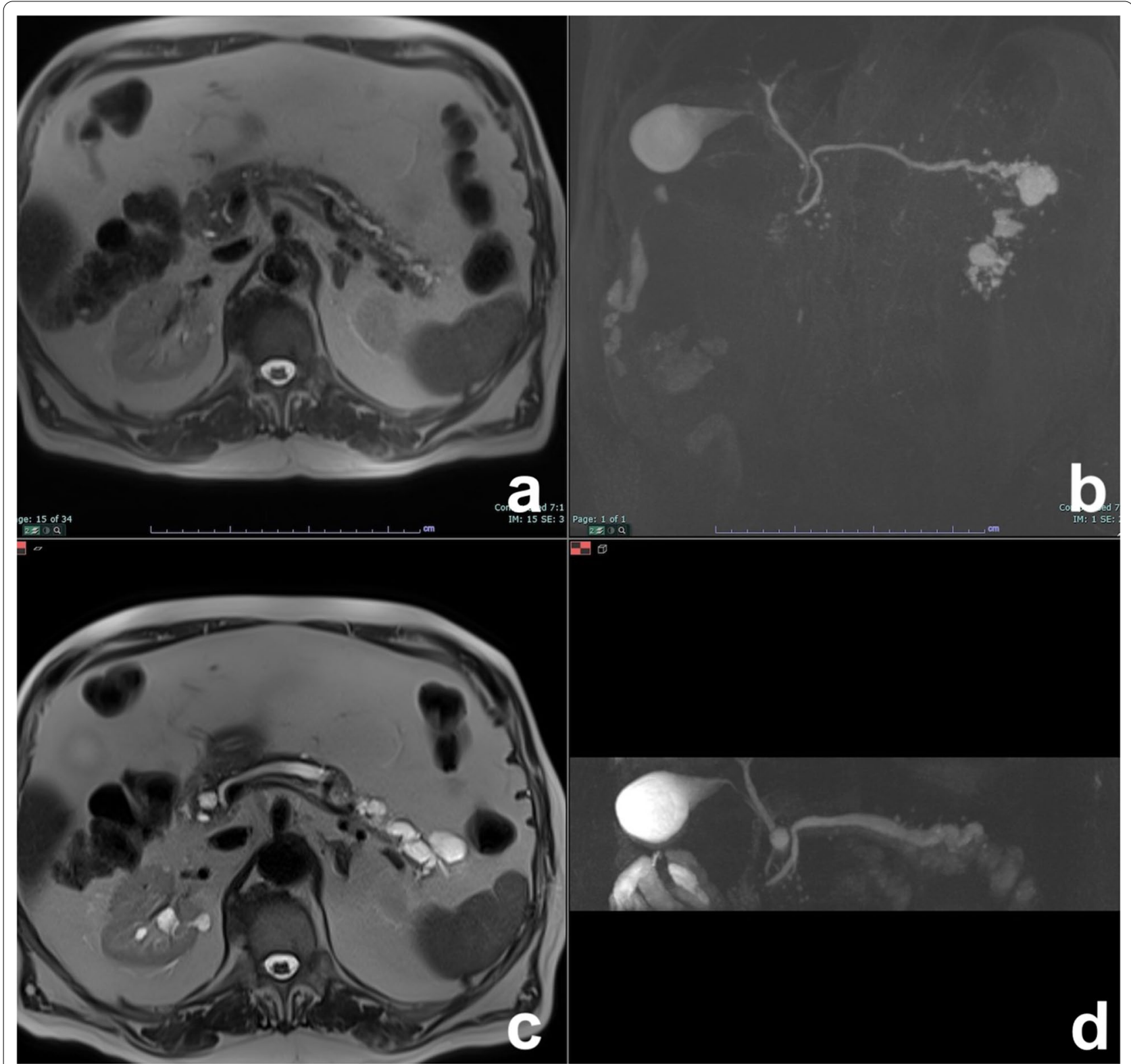

Fig. 16 Evolution of a mixed MD-IPMN. At T2W (a) and MRCP (b), a dilatation of a branch duct in the tail of the pancreas with a slight enlargement of the MPD in the tail. After 5 years, a diffuse dilatation of the MPD is appreciable

Many other guidelines for management of IPMN or CPNs including IPMN have been published [24], but none of them is complete, mainly because knowledge of natural history of these tumors is not perfect yet [63].

In our opinion, the IAP guidelines, in which treatment and follow-up strategies are decided on the basis of the presence of "high-risk stigmata" and "worrisome features," and the European guidelines are the most comprehensive and practical for decision making; however, the final decision on treatment should be individualized and should depend not only on the risk of malignancy and on the presence of symptoms, but also on patient's life expectancy, comorbidities, operative risk, cyst location and extent of surgery.

In particular, it is important to remember that the only possible treatment is surgery, and even in highvolume centers, risk of mortality and morbidity for pancreatic surgery is high (up to 3 and $30 \%$, respectively) [72]; moreover, according to a recent study on risks for mortality in 1800 patients with asymptomatic 


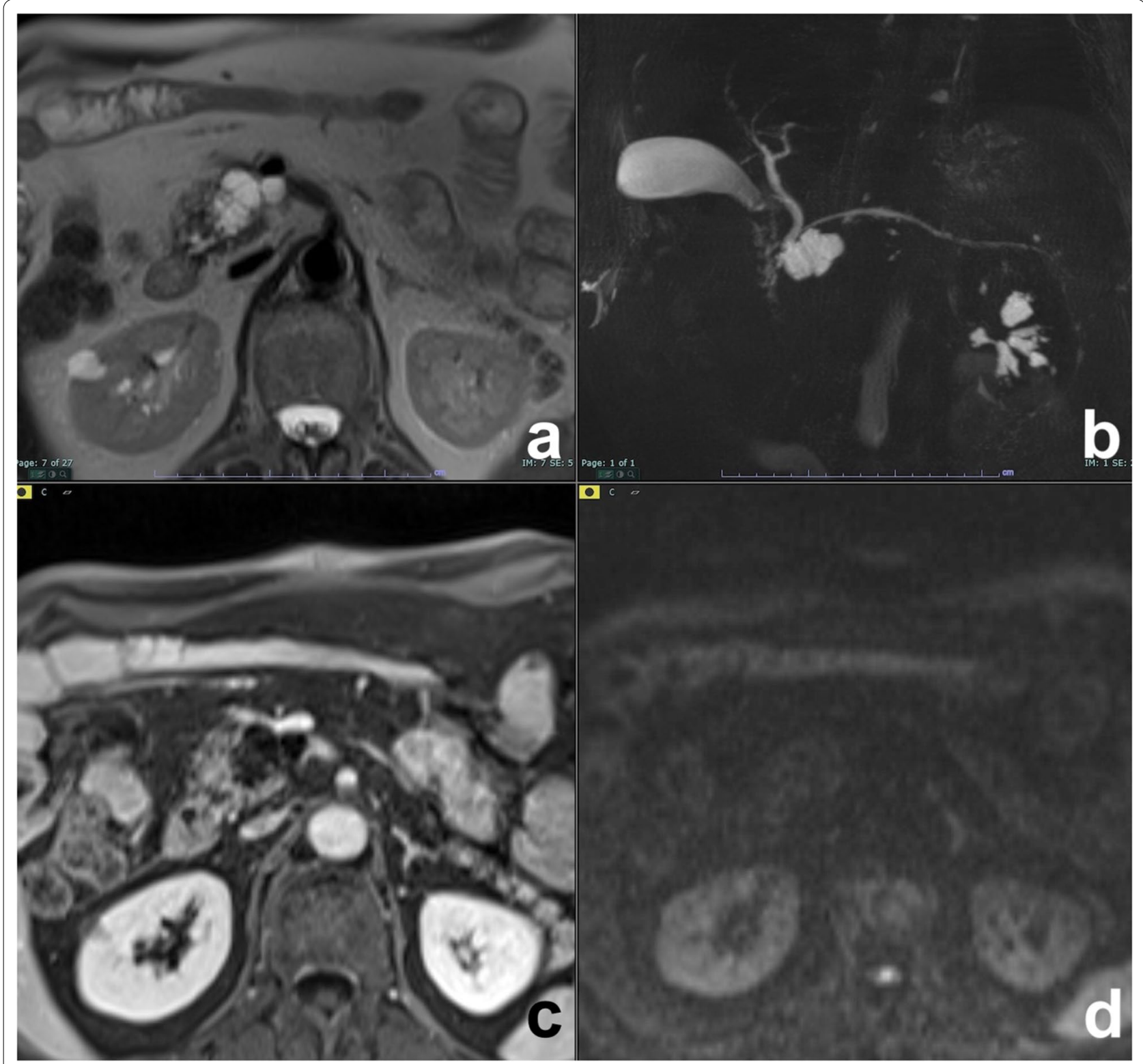

Fig. 17 Unifocal BD-IPMN. On T2w (a) and MRCP (b), a cystic lesion with tiny septa is appreciable in the head of the pancreas. At T1w after paramagnetic contrast media injection (c), the septa show a slight enhancement. At DWI b 800 (d), no restriction diffusion can be observed

CPL [73], comorbidity is the major cause of mortality, and only in patients with "high-risk cysts" (considered those with size $>3 \mathrm{~cm}$ and main pancreatic duct dilatation) and "low-risk comorbidities," the risk of pancreatic cancer mortality approaches that of non-pancreatic cancer mortality. Thus, patients-related factors such as age, life expectancy and comorbidities should strongly impact on clinical decision making.

All patients "fit for surgery" with high-risk stigmata should undergo surgery [64, 71], but also young patients fit for surgery with worrisome features should undergo to resection, due to their long life expectancy and higher cumulative risk of malignant progression of IPMN or de novo development of PDAC.

Patients fit for surgery without high-risk stigmata or worrisome features will undergo surveillance both for early detection of malignant progression of IPMN and for early detection of concomitant PDAC; surveillance for PDAC is needed also in patients who have undergone resection, even for noninvasive IPMN [63, 74].

The interval and period of surveillance are still controversial: IAP guidelines recommend intervals stratified 


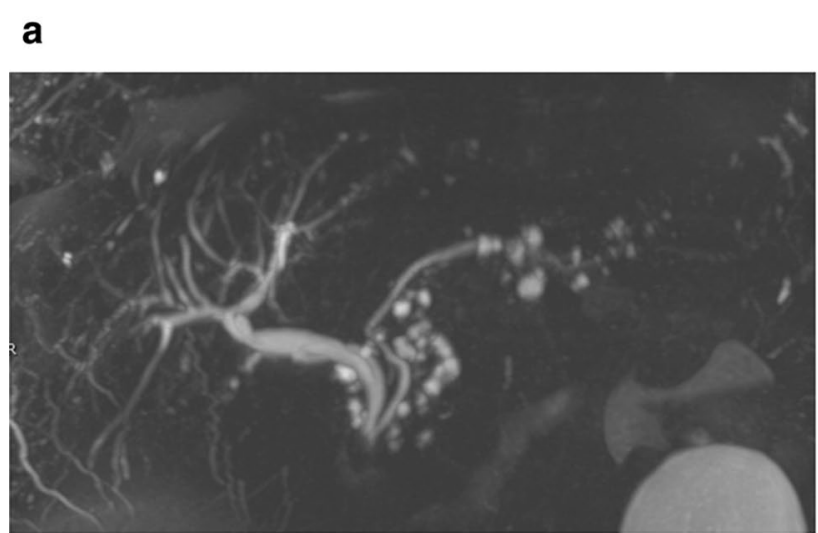

\section{b}

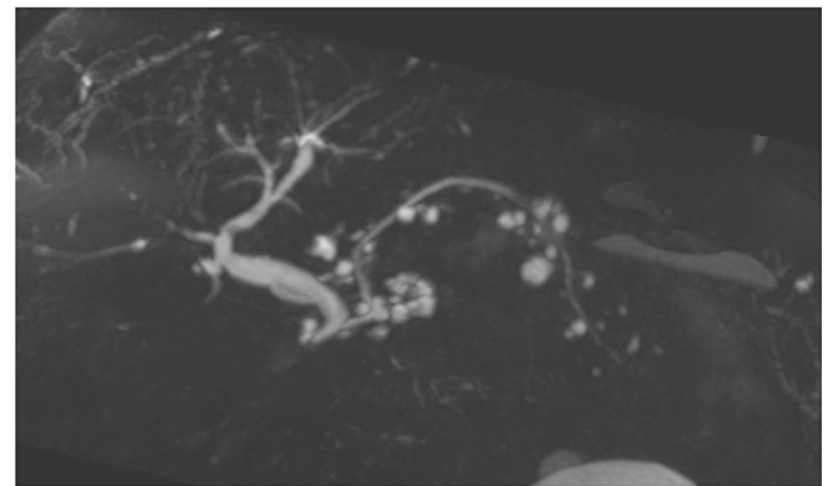

Fig. 18 Multifocal BD-IPMN. At MRCP in coronal (a) and axial (b) projection, several round dilatations of branch ducts in thigh connection with the MPD can be appreciable

Table 3 Fukuoka consensus guidelines, 2012 (intraductal papillary mucinous neoplasm—IPMN_and mucinous cystic neoplasm— MCN) and 2017 (intraductal papillary mucinous neoplasm-IPMN)

\section{Fukuoka consensus}

\section{Worrisome features}

Cyst size $>3 \mathrm{~cm}$

Thickened/enhancing cyst walls MPD dilatation of 5-9 $\mathrm{mm}$

Non-enhancing mural nodules

Abrupt changes in the MPD caliber with distal pancreatic atrophy

Lymphadenopathy

History of pancreatitis

\section{Revised Fukuoka consensus}

Worrisome features High-risk stigmata

Cyst size $>3 \mathrm{~cm}$

Thickened/enhancing cyst walls

MPD dilatation of 5-9 $\mathrm{mm}$

Enhancing mural nodules $<5 \mathrm{~mm}$

Abrupt changes in the MPD caliber with

Distal pancreatic atrophy

Lymphadenopathy

Rapid rate of cyst growth $>5 \mathrm{~mm} / 2$ years

Elevated serum level of CA 19.9

Table 4 Follow-up strategies according to Fukuoka consensus guidelines

\section{Diameter of the greatest cyst}

$<1 \mathrm{~cm}$

$1-2 \mathrm{~cm}$

$2-3 \mathrm{~cm}$

$>3 \mathrm{~cm}$

\section{Follow-up}

CT/MRI in 6 months

Then every 2 years if no change

$\mathrm{CT} / \mathrm{MRI}$ in 6 months

Yearly for 2 years

Then lengthen interval if no change

EUS in 3-6 months

Then lengthen interval if no change alternating MRI with EUS

Consider surgery in young fit Patients with need for prolonged survival Close surveillance alternating MRI with EUS every 3-6 months

Strongly consider surgery in young fit patients 
Table 5 European guidelines criteria for resection 2018

\begin{tabular}{ll}
\hline $\mathbf{2 0 1 8}$ European guidelines & \\
\hline Absolute indications & Relative indications \\
\hline Positive cytology for malignancy/HGD & Growth rate $\geq 5 \mathrm{~mm} /$ year \\
Solid mass & Serum CA 19.9 $>37 \mathrm{U} / \mathrm{ml}$ in absence of jaundice \\
Tumor-related jaundice & MPD diameter between 5 and 9.9 \\
Enhancing mural nodules $\geq 5 \mathrm{~mm}$ & Cyst diameter $\geq 40 \mathrm{~mm}$ \\
MPD dilatation $\geq 10 \mathrm{~mm}$ & Enhancing mural nodule $<5 \mathrm{~mm}$ \\
& New onset of diabetes mellitus \\
& Acute pancreatitis caused by IPMN
\end{tabular}

by the cyst size, which can be lengthened after 2 years of stability; other guidelines recommend discontinuation after 2 years (American College of Radiology 2010) or 5 years (American Gastroenterology Association 2015) of stability [63], because, according to the authors, the small risk of malignant progression in stable cysts is outweighed by the costs of surveillance. However, according to Tanaka [63] discontinuing or lengthening surveillance may be dangerous because of the long-lasting risk of concomitant PDAC in patients with IPMN [63], and even a 6-month interval might be insufficient for an early diagnosis of PDAC $[75,76]$. In particular, patients with IPMN or patients who have been resected for IPMN need follow-up not only to early detect malignant degeneration of IPMN, but also to early detect concomitant PDAC, synchronous or metachronous.

Tada et al. [77], in a study on 197 patients with IPMN and other CPLs, observed that this population is "at high risk" of developing a PDAC, with an incidence of pancreatic cancer 22.5 times higher than expected mortality in general population; Miyasaka et al. [74], on a surveillance study on 195 patients who underwent partial pancreatectomy for IPMN, demonstrated that the risk of malignant progression of IPMN and of developing a PDAC may even rise after 5 years; and $\mathrm{He} \mathrm{J}$ et al. [78], on a study on a surveillance 130 patients in follow-up after pancreatic resection for IPMN, observed that the risk of developing a new IPMN requiring surgery or PDAC was $0 \%, 7 \%$ and $38 \%$, respectively, at 1,5 and 10 years.

Thus, all patients with IPMN who are fit for treatment, even those who have been resected for a noninvasive IPMN, need indefinite surveillance which should be stopped only when the patient becomes unfit for surgery. The interval of follow-up is still a matter of discussion; however, it should be kept in mind that a long (more than 1 year) interval is dangerous, as after such a long followup a new appearing lesion could already be unresectable at diagnosis [75].
While follow-up strategies for early detection of malignant degeneration of IPMN are well established on the basis of dimensional criteria, there are not screening criteria for early detection of concomitant PDAC yet. Different studies have demonstrated that IPMN is a major risk factor for PDAC (having PDAC 1\% annual prevalence of concomitance with IPMN) [79-82], but some patients with IPMN have even higher risk of developing PDAC, in particular patients $>70$ years and women $[79,82]$, patients with benign gastric-type IPMN without guanine nucleotide-binding protein alpha-stimulating (GNAS) mutations [82-84] and patients with IPMN and family history of PDAC especially with affected firstdegree relatives $[82,85,86]$. This group of patients should be considered at higher risk, and tailored follow-up strategies should be applied.

A short interval follow-up should be considered in these categories, in order to avoid that a PDAC arising in the interval between two studies becomes unresectable, in particularly in young patients, as the cumulative risk of developing PDAC can rise up to $38 \%$ at 10 years [78]. As suggested by $\mathrm{He}$ et al. [78], cross-sectional imaging (MDCT or MRI) every 6 to 12 months for the first 5 years and annually thereafter should be offered.

This follow-up strategy of course rises the big issue of a large number of asymptomatic patients overloading radiological centers, due to the high prevalence of CPLs, especially in older patients (up to 40\%) [82, 87]. To overcome this problem, an effort from multidisciplinary teams should be made in order to select patients who should undergo such a strict follow-up, not only on the basis of the potential malignant degeneration and de novo PDAC development, but also on the basis of patients' comorbidities and operative risk. Moreover, as the best modality to follow these patients is MRI, and since it is "time-consuming," fast pancreas-dedicated MRI protocols should be considered.

Some authors have already established that a short MR protocol provides information equivalent to a more 
time-consuming and costly comprehensive protocol in the detection of significant changes of cystic lesions as well as in the detection of mural nodules [88]. Moreover, in our experience (analysis in a series of 200 patients, data still not published) with a fast non-contrast protocol (compared with a full MR protocol with contrast media injection), it is possible to detect a small PDAC with a sensitivity and negative predictive value ranging from 92\%-95\% and 98\%-99\%, respectively.

\section{Endoscopic ultrasound-fine needle aspiration (EUS-FNA) and cyst fluid analysis and cyst-wall biopsy}

Considering the morbidity and mortality rate of pancreatic surgery $[72,73]$, it should be very important to obtain correct preoperative diagnosis and correct preoperative evaluation of the grade of malignancy of CPLs.

A combination of the clinical history, gender and imaging findings sometimes is not enough in order to obtain a correct diagnosis. In such situations, EUS, EUS-FNA, EUS-TTNB can be helpful diagnostic tools: cytology, cyst fluid analyses of carcinoembryonic antigen (CEA), amylase and molecular biomarkers as well as cyst wall biopsy, allow distinction of mucinous vs non-mucinous cystic neoplasms and permit identification of the specific histological types $[9,63]$.

Analysis of cyst fluid obtained under EUS guidance permits the measurement of tumor markers and pancreatic enzymes, makes possible cytologic evaluation and allows to perform molecular DNA sequencing techniques which may help to distinguish serous versus mucinous cysts and to grade the epithelium of mucinous cysts [63].

Although accuracy of morphological findings of EUS in the differential diagnosis of PCLs is extremely operator dependent, ranging from 40 to $96 \%$ [89], more invasive techniques such as FNA for fluid analysis and cytology or endoscopic ultrasound-through the needle biopsy (EUS-TTNB) can increase significantly the diagnostic performance.

EUS is operator dependent and cyst fluid analysis very complex, so EUS with FNA and cytological and molecular analysis should be performed only in reference centers.

Elevated CEA allows distinction between mucinous and non-mucinous cysts (but not malignant versus nonmalignant cysts), and a cutoff value of $\geq 192-200 \mathrm{ng} / \mathrm{ml}$ is $79 \%$ accurate for the diagnosis of a mucinous cyst with moderate sensitivity $(73 \%)$ and specificity $(84 \%)$, while a cutoff value of $<5 \mathrm{ng} / \mathrm{ml}$ is the most valuable to exclude mucinous neoplasm and suspect SCN [90, 91].

Elevated amylase levels in the cyst fluid are highly specific for pseudocyst, and a cutoff value of $250 \mathrm{U} / \mathrm{L}$ is associated with the diagnosis of pseudocyst with a sensitivity of $44 \%$ and a specificity of $98 \%$, and thus, amylase level $<250 \mathrm{U} / \mathrm{L}$ virtually excludes the presence of a pseudocyst [90, 92].

Cytological analysis of cyst's fluid obtained under EUS guidance has good specificity (up to $83 \%$ ) for diagnosing mucinous cysts, but very low sensitivity $(<50 \%)$ because of the small amount of cells dispersed in the fluid, and moreover is difficult to interpret [91, 93-95].

Molecular analysis of the cyst fluid for diagnosis is still evolving; detection of KRAS gene mutation more accurately supports the diagnosis of a mucinous but not necessarily a malignant cyst (96\% Sp) [71, 96-98], while identification of GNAS gene mutation may be helpful in distinguishing significant mucinous cysts from indolent cysts that could be conservatively managed $[99,100]$.

Cytology brush is not indicated because of the high rate of complications (8-20\%, included death) and of technical failure (27\%) with conflicting results [90, 101-103].

Tissue acquisition is the ideal method for diagnosis and risk stratification in CPLs. Barresi et al. [95], in a multicenter study on 56 Patients with CPLs, demonstrated that EUS-TTNB is a promising technique that provides specimens adequate for histological diagnosis in $83.9 \%$ of cases with acceptable rate of adverse events (16\%), all of which classified as mild.

\section{New imaging applications}

Distinguishing invasive from noninvasive IPMNs preoperatively remains challenging. Khoury et al. in a cohort of 478 pancreatectomies performed for IPMN in a single year in over 100 US institutions found that invasive carcinoma or high-grade dysplasia was identified only in $23 \%$ of resected lesions, so improved biomarkers are needed to aid in surgical selection [104].

In the last years, different studies have demonstrated that quantitative imaging and radiomics applied to MDCT pancreatic protocols can provide markers for reliably discriminating benign from malignant IPMNs (low- and intermediate-grade dysplasia versus highgrade dysplasia and invasive carcinoma), thus allowing objective risk stratification of these lesions [105-108]. In particular, in the most robust of these studies conducted on MDCT images of 408 resected patients with IPMN, Tobaly et al. [108] found that in the training cohort, 85 radiomics features were significantly different between patients with benign and malignant IPMNs; they also demonstrated that the multivariate model of radiomics only differentiated benign from malignant tumors in training cohort with an area under the ROC curve (AUC) of 0.84 , Se of 0.82 , Sp of 0.74 and in the external validation cohort with an AUC of 0.71, Se of 0.69 and Sp of 0.57 . 
MRI-based radiomics is much more difficult because MRI suffers from less standardization than CT with a large variety in the multiple parameters related to scanner properties, acquisition settings and image processing that could hamper radiomics analysis [108]. However, in a retrospective series of 248 patients with surgically confirmed IPMN, Jeon et al. among texture variables found 7 significant predictors of malignancy $(p<0.05), 2$ of which confirmed to be significant predictors of malignancy also at multivariate analysis. Moreover, when adding texture variable to MRI findings, diagnostic performance for predicting malignant IPMN improved from 0.80 and 0.78 to 0.85 in both reviewers $(p<0.05)$ [109].

\section{Conclusions}

CPLs are a heterogeneous group of frequently detected lesions with different malignant potential and different prognosis, requiring different therapeutic and follow-up strategies; therefore, it is fundamental to correctly characterize them. MRI with MRCP sequence is the most useful technique to detect imaging features helpful for lesion characterization.

Differential diagnosis of these cysts is based on the presence of communication or not with MPD, on their morphological features, on their location as well as on the basis of clinical history and demographic data.

Once pseudocyst and WON have been excluded, a CPL communicating with MPD is an IPMN, while if not communicating could be a SCN, a MCN or a SPN and differential diagnosis, certain or presumptive, can be done on the basis of demographic data, location of the lesion and its morphological appearance.

In some cases, differential diagnosis is still challenging and may require EUS-FNA and/or cyst-wall biopsy.

MCNs and IPMN with high-risk stigmata must be resected if patients are fit for surgery; in patients with long life expectancy and IPMN with worrisome features, resection is suggested.

Patients with IPMN without high-risk stigmata and patients who have been resected for an IPMN need follow-up till they are fit for surgery, in order to early detect malignant degeneration or concomitant pancreatic ductal adenocarcinoma.

When the diagnosis of a CPL remains indeterminate, in our opinion it should be managed as an IPMN, as they are the most frequent CPLs with a potential malignant behavior.

New imaging applications, with quantitative analysis thanks to texture analysis, have the potential to help to better stratify IPMNs, thus allowing a more correct management.

\section{Abbreviations}

ACCN: Acinar cell cystic neoplasm; CEA: Carcinoembryonic antigen; CPLs: Cystic pancreatic lesions; CPN: Cystic pancreatic neoplasm; CPNETs: Cystic pancreatic neuroendocrine tumors; ERCP: Endoscopic retrograde cholangiopancreatography; EUS: Endoscopic ultrasound; EUS-FNA: Endoscopic ultrasound-fine needle aspiration; EUS-TTNB: Endoscopic ultrasound-through the needle biopsy; GNAS: Guanine nucleotide binding protein, alpha stimulating; IPMN: Intraductal papillary mucinous neoplasm; MCN: Mucinous cystic neoplasm; MDCT: Multidetector computed tomography; MPD: Main pancreatic duct; MPR: Multiplanar reconstruction; MRCP: Magnetic resonance cholangiopancreatography; MRI: Magnetic resonance imaging; PDAC: Pancreatic ductal adenocarcinoma; SCN: Serous cystic neoplasm; SPN: Solid pseudopapillary neoplasm; US: Ultrasound; WON: Walled-off necrosis.

\section{Authors' contributions}

All authors made a substantial contribution. All authors drafted the work and substantively revised it. All have approved the submitted version.

\section{Funding}

Not applicable.

\section{Availability of data and materials}

Data sharing is not applicable to this article as no datasets were generated or analyzed during the current study.

\section{Declarations}

Ethics approval and consent to participate

Not applicable.

\section{Consent for publication}

Images are entirely unidentifiable, and there are no details on individuals reported within the manuscript.

\section{Competing interests}

The authors declare that they have no competing interests.

\section{Author details}

'Department of Radiology, Regional Hospital Ca'Foncello, Piazza Ospedale 1, 3100 Treviso, Italy. ${ }^{2}$ Department of Radiology and Nuclear Medicine, Erasmus MC, Rotterdam, The Netherlands.

Received: 3 March 2021 Accepted: 17 July 2021

Published online: 10 August 2021

\section{References}

1. Zhang XM, Mitchell DG, Dohke M, Holland GA, Parker L (2002) Pancreatic cysts: depiction on single-shot fast spin-echo MR images. Radiology 223:547-553. https://doi.org/10.1148/radiol.2232010815

2. Ks S, Te F, Ra D, et al (2004) Cystic pancreatic neoplasms: observe or operate. Ann Surg https://pubmed.ncbi.nlm.nih.gov/15082969/. Accessed 10 Feb 2021

3. Laffan TA, Horton KM, Klein AP et al (2008) Prevalence of unsuspected pancreatic cysts on MDCT. AJR Am J Roentgenol 191:802-807. https:// doi.org/10.2214/AJR.07.3340

4. de Jong K, Nio CY, Hermans JJ et al (2010) High prevalence of pancreatic cysts detected by screening magnetic resonance imaging examinations. Clin Gastroenterol Hepatol 8:806-811. https://doi.org/10. 1016/j.cgh.2010.05.017

5. Lee KS, Sekhar A, Rofsky NM, Pedrosa I (2010) Prevalence of incidental pancreatic cysts in the adult population on MR imaging. Am J Gastroenterol 105(9):2079-2084

6. Brugge WR (2015) Diagnosis and management of cystic lesions of the pancreas. J Gastrointest Oncol 6:375-388. https://doi.org/10.3978/j.issn. 2078-6891.2015.057

7. Lanke G, Lee JH (2020) Similarities and differences in guidelines for the management of pancreatic cysts. World J Gastroenterol 26:1128-1141. https://doi.org/10.3748/wjg.v26.i11.1128 
8. Nougaret S, Mannelli L, Pierredon MA, Schembri V, Guiu B (2016) Cystic pancreatic lesions: from increased diagnosis rate to new dilemmas. Diagn Interv Imaging 97:1275-1285. https://doi.org/10.1016/j.diii.2016. 08.017

9. European Study Group on Cystic Tumours of the Pancreas (2018) European evidence-based guidelines on pancreatic cystic neoplasms. Gut 67:789-804. https://doi.org/10.1136/gutjnl-2018-316027

10. van Huijgevoort NC, Del Chiaro M, Wolfgang CL, van Hooft JE, Besselink MG (2019) Diagnosis and management of pancreatic cystic neoplasms: current evidence and guidelines. Nat Rev Gastroenterol Hepatol 16:676-689. https://doi.org/10.1038/s41575-019-0195-x

11. Foster BR, Jensen KK, Bakis G et al (2016) Revised Atlanta classification for acute pancreatitis: a pictorial essay. Radiographics 36:675-687. https://doi.org/10.1148/rg.2016150097

12. Adler DG, Siddiqui AA (2016) What's in a name? Pancreatic pseudocysts, walled-off necrosis, and pancreatic fluid collections. Endosc Ultrasound 5:215-217. https://doi.org/10.4103/2303-9027.187837

13. Sainani NI, Saokar A, Deshpande V, Castillo CFD, Hahn P, Sahani DV (2009) Comparative performance of MDCT and MRI with MR cholangiopancreatography in characterizing small pancreatic cysts. AJR Am J Roentgenol 193(3):722-731

14. Lee HJ, Kim MJ, Choi JY, Hong HS, Kim KA (2011) Relative accuracy of $\mathrm{CT}$ and MRI in the differentiation of benign from malignant pancreatic cystic lesions. Clin Radiol. https://pubmed.ncbi.nlm.nih.gov/21356393/. Accessed 8 Feb 2021

15. Kim JH, Eun HW, Park HJ, Hong SS, Kim YJ (2012) Diagnostic performance of MRI and EUS in the differentiation of benign from malignant pancreatic cyst and cyst communication with the main duct. Eur $\mathrm{J}$ Radiol. https://pubmed.ncbi.nlm.nih.gov/22227264/. Accessed 8 Feb 2021

16. Sahani DV, Sainani NI, Blake MA, Crippa S, Mino-Kenudson M, delCastillo CF (2011) Prospective evaluation of reader performance on MDCT in characterization of cystic pancreatic lesions and prediction of cyst biologic aggressiveness. AJR Am J Roentgenol 197:W53-61. https:// doi.org/10.2214/AJR.10.5866

17. Banks PA, Bollen TL, Dervenis C et al (2013) Classification of acute pancreatitis-2012: revision of the Atlanta classification and definitions by international consensus. Gut 62:102-111. https://doi.org/10.1136/ gutjnl-2012-302779

18. Fernández-del Castillo C, Targarona J, Thayer SP, Rattner DW, Brugge WR, Warshaw AL (2003) Incidental pancreatic cysts: clinicopathologic characteristics and comparison with symptomatic patients. Arch Surg Chic III 1960 138:427-423; discussion 433-434. https://doi.org/10.1001/ archsurg. 138.4.427

19. Cui ML, Kim KH, Kim HG et al (2014) Incidence, risk factors and clinical course of pancreatic fluid collections in acute pancreatitis. Dig Dis Sci 59:1055-1062. https://doi.org/10.1007/s10620-013-2967-4

20. Macari M, Finn ME, Bennett GL et al (2009) Differentiating pancreatic cystic neoplasms from pancreatic pseudocysts at MR imaging: value of perceived internal debris. Radiology 251:77-84. https://doi.org/10. 1148/radiol.2511081286

21. Al-Salem AH (2014) Congenital pancreatic cysts. In: Al-Salem AH (ed) An illustrated guide to pediatric surgery. Springer, Cham, pp 309-313

22. Kim YS, Cho JH (2015) Rare nonneoplastic cysts of pancreas. Clin Endosc 48:31-38. https://doi.org/10.5946/ce.2015.48.1.31

23. Ren F, Zuo C, Chen G et al (2013) Pancreatic retention cyst: multimodality imaging findings and review of the literature. Abdom Imaging 38:818-826. https://doi.org/10.1007/s00261-012-9976-1

24. Megibow AJ, Baker ME, Morgan DE et al (2017) Management of incidental pancreatic cysts: a white paper of the ACR incidental findings committee. J Am Coll Radiol 14:911-923. https://doi.org/10.1016/j. jacr.2017.03.010

25. Del Chiaro M, Verbeke C, Salvia R et al (2013) European experts consensus statement on cystic tumours of the pancreas. Dig Liver Dis 45:703-711. https://doi.org/10.1016/j.dld.2013.01.010

26. Jais B, Rebours V, Malleo G et al (2016) Serous cystic neoplasm of the pancreas: a multinational study of 2622 patients under the auspices of the International Association of Pancreatology and European Pancreatic Club (European Study Group on Cystic Tumors of the Pancreas). Gut 65:305-312. https://doi.org/10.1136/gutjnl-2015-309638
27. Horvath KD, Chabot JA (1999) An aggressive resectional approach to cystic neoplasms of the pancreas. Am J Surg 178:269-274

28. Bassi C, Salvia R, Molinari E, Biasutti C, Falconi M, Pederzoli P (2003) Management of 100 consecutive cases of pancreatic serous cystadenoma: wait for symptoms and see at imaging or vice versa? World J Surg 27:319-323. https://doi.org/10.1007/s00268-002-6570-7

29. Neumann HP, Dinkel E, Brambs H et al (1991) Pancreatic lesions in the von Hippel-Lindau syndrome. Gastroenterology 101:465-471

30. Choi J-Y, Kim M-J, Lee JY et al (2009) Typical and atypical manifestations of serous cystadenoma of the pancreas: imaging findings with pathologic correlation. AJR Am J Roentgenol 193:136-142. https:// doi.org/10.2214/AJR.08.1309

31. Shah AA, Sainani NI, Kambadakone AR et al (2009) Predictive value of multi-detector computed tomography for accurate diagnosis of serous cystadenoma: radiologic-pathologic correlation. World J Gastroenterol 15:2739-2747

32. Wang GX, Wang ZP, Chen HL, Zhang D, Wen L (2020) Discrimination of serous cystadenoma from mucinous cystic neoplasm and branch duct intraductal papillary mucinous neoplasm in the pancreas with CT. Abdom Radiol (NY) 45:2772-2778. https://doi.org/10.1007/ s00261-020-02664-7

33. Kim SY, Lee JM, Kim SH et al (2006) Macrocystic neoplasms of the pancreas: CT differentiation of serous oligocystic adenoma from mucinous cystadenoma and intraductal papillary mucinous tumor. AJR Am J Roentgenol 187:1192-1198. https://doi.org/10.2214/AJR. 05.0337

34. Park HS, Kim SY, Hong S-M et al (2016) Hypervascular solid-appearing serous cystic neoplasms of the pancreas: Differential diagnosis with neuroendocrine tumours. Eur Radiol 26:1348-1358. https://doi.org/ 10.1007/s00330-015-3961-3

35. Chu LC, Singhi AD, Haroun RR et al (2017) The many faces of pancreatic serous cystadenoma: Radiologic and pathologic correlation. Diagn Interv Imaging 98:191-202. https://doi.org/10.1016/j.diii.2016. 08.005

36. Tseng JF, Warshaw AL, Sahani DV, Lauwers GY, Rattner DW, Fernandezdel Castillo C (2005) Serous cystadenoma of the pancreas: tumor growth rates and recommendations for treatment. Ann Surg 242:413419; discussion 419-421

37. Buetow PC, Rao P, Thompson LD (1998) From the archives of the AFIP. Mucinous cystic neoplasms of the pancreas: radiologic-pathologic correlation. Radiographics 18:433-449. https://doi.org/10.1148/radiograph ics.18.2.9536488

38. Zamboni G, Scarpa A, Bogina G et al (1999) Mucinous cystic tumors of the pancreas: clinicopathological features, prognosis, and relationship to other mucinous cystic tumors. Am J Surg Pathol 23:410-422

39. Goh BKP, Tan Y-M, Chung Y-FA et al (2006) A review of mucinous cystic neoplasms of the pancreas defined by ovarian-type stroma: clinicopathological features of 344 patients. World J Surg 30:2236-2245. https://doi.org/10.1007/s00268-006-0126-1

40. Crippa S, Salvia R, Warshaw AL et al (2008) Mucinous Cystic Neoplasm of the Pancreas is Not an Aggressive Entity. Ann Surg 247:571-579. https://doi.org/10.1097/SLA.0b013e31811f4449

41. Cohen-Scali F, Vilgrain V, Brancatelli G et al (2003) Discrimination of unilocular macrocystic serous cystadenoma from pancreatic pseudocyst and mucinous cystadenoma with CT: initial observations. Radiology 228:727-733. https://doi.org/10.1148/radiol.2283020973

42. Sahani DV, Kadavigere R, Saokar A, Fernandez-del Castillo C, Brugge WR, Hahn PF (2005) Cystic pancreatic lesions: a simple imagingbased classification system for guiding management. Radiographics 25:1471-1484. https://doi.org/10.1148/rg.256045161

43. Di Paola V, Manfredi R, Mehrabi S et al (2016) Pancreatic mucinous cystoadenomas and cystoadenocarcinomas: differential diagnosis by means of MRI. Br J Radiol. https://doi.org/10.1259/bjr.20150536

44. Al Efishat M, Allen PJ (2016) Therapeutic approach to cystic neoplasms of the pancreas. Surg Oncol Clin N Am 25:351-361. https:// doi.org/10.1016/j.soc.2015.11.006

45. Coleman KM, Doherty MC, Bigler SA (2003) Solid-pseudopapillary tumor of the pancreas. Radiographics 23:1644-1648. https://doi.org/ 10.1148/rg.236035006

46. Buetow PC, Buck JL, Pantongrag-Brown L, Beck KG, Ros PR, Adair CF (1996) Solid and papillary epithelial neoplasm of the pancreas: 
imaging-pathologic correlation on 56 cases. Radiology 199:707-711. https://doi.org/10.1148/radiology.199.3.8637992

47. Seo H-E, Lee M-K, Lee Y-D et al (2006) Solid-pseudopapillary Tumor of the Pancreas. J Clin Gastroenterol 40:919-922. https://doi.org/10. 1097/01.mcg.0000225671.91722.10

48. Ventriglia A, Manfredi R, Mehrabi S et al (2014) MRI features of solid pseudopapillary neoplasm of the pancreas. Abdom Imaging 39:1213-1220. https://doi.org/10.1007/s00261-014-0169-y

49. Naar L, Spanomichou DA, Mastoraki A, Smyrniotis V, Arkadopoulos N (2017) Solid pseudopapillary neoplasms of the pancreas: a surgical and genetic enigma. World J Surg 41:1871-1881. https://doi.org/10. 1007/s00268-017-3921-y

50. Zamboni G, Terris B, Scarpa A et al (2002) Acinar cell cystadenoma of the pancreas: a new entity? Am J Surg Pathol 26:698-704. https://doi. org/10.1097/00000478-200206000-00002

51. Singhi AD, Norwood S, Liu T-C et al (2013) Acinar cell cystadenoma of the pancreas: a benign neoplasm or non-neoplastic ballooning of acinar and ductal epithelium? Am J Surg Pathol 37:1329-1335. https://doi.org/10.1097/PAS.0b013e3182a1ad72

52. Wang G, Ji L, Qu F-Z et al (2016) Acinar cell cystadenoma of the pancreas: A retrospective analysis of ten-year experience from a single academic institution. Pancreatology 16:625-631. https://doi.org/10. 1016/j.pan.2016.03.020

53. Delavaud C, d'Assignies G, Cros J et al (2014) CT and MR imaging of multilocular acinar cell cystadenoma: comparison with branch duct intraductal papillary mucinous neoplasia (IPMNs). Eur Radiol 24:2128-2136. https://doi.org/10.1007/s00330-014-3248-0

54. Bordeianou L, Vagefi PA, Sahani D et al (2008) Cystic pancreatic endocrine neoplasms: a distinct tumor type? J Am Coll Surg 206:11541158. https://doi.org/10.1016/j.jamcollsurg.2007.12.040

55. Singhi AD, Chu LC, Tatsas AD et al (2012) Cystic pancreatic neuroendocrine tumors: a clinicopathologic study. Am J Surg Pathol 36:1666-1673. https://doi.org/10.1097/PAS.0b013e31826a0048

56. Zhu J-K, Wu D, Xu J-W et al (2019) Cystic pancreatic neuroendocrine tumors: a distinctive subgroup with indolent biological behavior? A systematic review and meta-analysis. Pancreatology 19:738-750. https://doi.org/10.1016/j.pan.2019.05.462

57. Kalb B, Sarmiento JM, Kooby DA, Adsay NV, Martin DR (2009) MR imaging of cystic lesions of the pancreas. Radiographics 29:1749-1765. https://doi.org/10.1148/rg.296095506

58. Cloyd JM, Kopecky KE, Norton JA et al (2016) Neuroendocrine tumors of the pancreas: Degree of cystic component predicts prognosis. Surgery 160:708-713. https://doi.org/10.1016/j.surg.2016.04.005

59. Paiella S, Marchegiani G, Miotto M et al (2018) Are cystic pancreatic neuroendocrine tumors an indolent entity results from a single-center surgical series. Neuroendocrinology 106:234-241. https://doi.org/10. 1159/000477849

60. Carr RA, Bletsis P, Roch AM et al (2019) Cystic pancreatic neuroendocrine tumors: a more favorable lesion? Pancreatology 19:372-376. https://doi.org/10.1016/j.pan.2019.01.017

61. Caglià P, Cannizzaro MT, Tracia A et al (2015) Cystic pancreatic neuroendocrine tumors: to date a diagnostic challenge. Int J Surg 21(Suppl 1):S44-49. https://doi.org/10.1016/i.jijsu.2015.04.087

62. Machado NO, Al Qadhi H, Al Wahibi K(2015) Intraductal papillary mucinous neoplasm of pancreas. North Am J Med Sci 7:160-175. https://doi. org/10.4103/1947-2714.157477

63. Tanaka M (2019) Clinical Management and surgical decision-making of IPMN of the pancreas. Methods Mol Biol. https://pubmed.ncbi.nlm.nih. gov/30378040/. Accessed 23 Feb 2021

64. Tanaka M, Fernández-del Castillo C, Adsay V et al (2012) International consensus guidelines 2012 for the management of IPMN and MCN of the pancreas. Pancreatology 12:183-197. https://doi.org/10.1016/j.pan. 2012.04.004

65. Kim JH, Hong SS, Kim YJ, Kim JK, Eun HW (2012) Intraductal papillary mucinous neoplasm of the pancreas: differentiate from chronic pancreatits by MR imaging. Eur J Radiol 81:671-676. https://doi.org/10.1016/j. ejrad.2011.01.066

66. Procacci C, Carbognin G, Biasiutti C, Guarise A, Ghirardi C, Schenal G (2001) Intraductal papillary mucinous tumors of the pancreas: spectrum of CT and MR findings with pathologic correlation. Eur Radiol 11:1939-1951. https://doi.org/10.1007/s003300100823
67. Lim JH, Lee G, Oh YL (2001) Radiologic spectrum of intraductal papillary mucinous tumor of the pancreas. Radiographics 21:323-337; discussion 337-340. https://doi.org/10.1148/radiographics.21.2.g01 mr01323

68. Procacci C, Megibow AJ, Carbognin G, et al (1999) Intraductal papillary mucinous tumor of the pancreas: a pictorial essay. Radiographics 19:1447-1463. https://doi.org/10.1 148/radiographics.19.6.g99no011447

69. Pilleul F, Rochette A, Partensky C, Scoazec JY, Bernard P, Valette PJ (2005) Preoperative evaluation of intraductal papillary mucinous tumors performed by pancreatic magnetic resonance imaging and correlated with surgical and histopathologic findings. J Magn Reson Imaging JMRI 21:237-244. https://doi.org/10.1002/jmri.20254

70. Buscarini E, Pezzilli R, Cannizzaro R et al (2014) Italian consensus guidelines for the diagnostic work-up and follow-up of cystic pancreatic neoplasms. Dig Liver Dis 46:479-493. https://doi.org/10.1016/j.dld.2013. 12.019

71. Tanaka M, Fernández-Del Castillo C, Kamisawa T et al (2017) Revisions of international consensus Fukuoka guidelines for the management of IPMN of the pancreas. Pancreatology 17:738-753. https://doi.org/10. 1016/j.pan.2017.07.007

72. Vollmer CM, Sanchez N, Gondek S, et al (2012) A root-cause analysis of mortality following major pancreatectomy. J Gastrointest Surg Off J Soc Surg Aliment Tract 16:89-102; discussion 102-103. https://doi.org/10. 1007/s11605-011-1753-x

73. Kwok K, Chang J, Duan L, Huang BZ, Wu BU (2017) Competing risks for mortality in patients with asymptomatic pancreatic cystic neoplasms: implications for clinical management. Am J Gastroenterol 112:13301336. https://doi.org/10.1038/ajg.2017.141

74. Miyasaka Y, Ohtsuka T, Tamura K et al (2016) Predictive factors for the metachronous development of high-risk lesions in the remnant pancreas after partial pancreatectomy for intraductal papillary mucinous neoplasm. Ann Surg 263:1180-1187. https://doi.org/10.1097/SLA.00000 00000001368

75. Tamura K, Ohtsuka T, Ideno N et al (2013) Unresectable pancreatic ductal adenocarcinoma in the remnant pancreas diagnosed during every-6-month surveillance after resection of branch duct intraductal papillary mucinous neoplasm: a case report. JOP 14:450-453. https:// doi.org/10.6092/1590-8577/1494

76. Mortenson T, Bigeh A, Chen Y et al (2017) Heterogeneous growth rates of pancreatic adenocarcinoma by retrospective analysis of CT imaging data. J Clin Oncol 35:248-248. https://doi.org/10.1200/JCO.2017.35.4 suppl.248

77. Tada M, Kawabe T, Arizumi M et al (2006) Pancreatic cancer in patients with pancreatic cystic lesions: a prospective study in 197 patients. Clin Gastroenterol Hepatol Off Clin Pract J Am Gastroenterol Assoc 4:1265-1270. https://doi.org/10.1016/j.cgh.2006.07.013

78. He J, Cameron JL, Ahuja N, et al (2013) Is it necessary to follow patients after resection of a benign pancreatic intraductal papillary mucinous neoplasm? J Am Coll Surg 216:657-665; discussion 665-667. https:// doi.org/10.1016/j.jamcollsurg.2012.12.026

79. Tanno S, Nakano Y, Sugiyama Y et al (2010) Incidence of synchronous and metachronous pancreatic carcinoma in 168 patients with branch duct intraductal papillary mucinous neoplasm. Pancreatology 10:173-178. https://doi.org/10.1159/000231982

80. Ingkakul T, Sadakari Y, lenaga J, Satoh N, Takahata S, Tanaka M (2010) Predictors of the presence of concomitant invasive ductal carcinoma in intraductal papillary mucinous neoplasm of the pancreas. Ann Surg 251:70-75. https://doi.org/10.1097/SLA.0b013e3181c5ddc3

81. Yamaguchi K, Kanemitsu S, Hatori T et al (2011) Pancreatic ductal adenocarcinoma derived from IPMN and pancreatic ductal adenocarcinoma concomitant with IPMN. Pancreas 40:571-580. https://doi.org/10. 1097/MPA.0b013e318215010c

82. Torisu Y, Takakura K, Kinoshita Y, Tomita Y, Nakano M, Saruta M (2019) Pancreatic cancer screening in patients with presumed branch-duct intraductal papillary mucinous neoplasms. World J Clin Oncol 10:67-74. https://doi.org/10.5306/wjco.v10.i2.67

83. Ideno N, Ohtsuka T, Matsunaga T et al (2015) Clinical significance of GNAS mutation in intraductal papillary mucinous neoplasm of the pancreas with concomitant pancreatic ductal adenocarcinoma. Pancreas 44:311-320. https://doi.org/10.1097/MPA.0000000000000258

84. Ideno N, Ohtsuka T, Kono Het al (2013) Intraductal papillary mucinous neoplasms of the pancreas with distinct pancreatic ductal 
adenocarcinomas are frequently of gastric subtype. Ann Surg 258:141-151. https://doi.org/10.1097/SLA.0b013e31828cd008

85. Nehra D, Oyarvide VM, Mino-Kenudson M et al (2012) Intraductal papillary mucinous neoplasms: does a family history of pancreatic cancer matter? Pancreatology 12:358-363. https://doi.org/10.1016/j.pan.2012. 05.011

86. Mandai K, Uno K, Yasuda K (2014) Does a family history of pancreatic ductal adenocarcinoma and cyst size influence the follow-up strategy for intraductal papillary mucinous neoplasms of the pancreas? Pancreas 43:917-921. https://doi.org/10.1097/MPA.00000000000000132

87. Moris M, Bridges MD, Pooley RA et al (2016) Association between advances in high-resolution cross-section imaging technologies and increase in prevalence of pancreatic cysts from 2005 to 2014. Clin Gastroenterol Hepatol Off Clin Pract J Am Gastroenterol Assoc 14:585-593. e3. https://doi.org/10.1016/j.cgh.2015.08.038

88. Pozzi-Mucelli RM, Rinta-Kiikka I, Wünsche K et al (2017) Pancreatic MRI for the surveillance of cystic neoplasms: comparison of a short with a comprehensive imaging protocol. Eur Radiol 27:41-50. https://doi.org/ 10.1007/s00330-016-4377-4

89. Lévy P, Rebours V (2018) The role of endoscopic ultrasound in the diagnosis of cystic lesions of the pancreas. Visc Med 34:192-196. https://doi. org/10.1159/000489242

90. Vilas-Boas F, Macedo G (2018) Pancreatic Cystic Lesions: New Endoscopic Trends in Diagnosis. J Clin Gastroenterol 52:13-19. https://doi. org/10.1097/MCG.0000000000000945

91. Brugge WR, Lewandrowski K, Lee-Lewandrowski E et al (2004) Diagnosis of pancreatic cystic neoplasms: a report of the cooperative pancreatic cyst study. Gastroenterology 126:1330-1336. https://doi. org/10.1053/j.gastro.2004.02.013

92. van der Waaij LA, van Dullemen HM, Porte RJ (2005) Cyst fluid analysis in the differential diagnosis of pancreatic cystic lesions: a pooled analysis. Gastrointest Endosc 62:383-389. https://doi.org/10.1016/s00165107(05)01581-6

93. Maker AV, Lee LS, Raut CP, Clancy TE, Swanson RS (2008) Cytology from pancreatic cysts has marginal utility in surgical decision-making. Ann Surg Oncol 15:3187-3192. https://doi.org/10.1245/s10434-008-0110-0

94. de Jong K, Poley JW, Van Hooft JE, Visser M, Bruno MJ, Fockens P (2011) Endoscopic ultrasound-guided fine-needle aspiration of pancreatic cystic lesions provides inadequate material for cytology and laboratory analysis: initial results from a prospective study. Endoscopy 43:585-590. https://doi.org/10.1055/s-0030-1256440

95. Barresi L, Crinò SF, Fabbri C et al (2018) Endoscopic ultrasound-throughthe-needle biopsy in pancreatic cystic lesions: a multicenter study. Dig Endosc 30:760-770. https://doi.org/10.1111/den.13197

96. Khalid A, McGrath KM, Zahid M et al (2005) The role of pancreatic cyst fluid molecular analysis in predicting cyst pathology. Clin Gastroenterol Hepatol Off Clin Pract J Am Gastroenterol Assoc 3:967-973. https://doi. org/10.1016/s1542-3565(05)00409-x

97. Khalid A, Zahid M, Finkelstein SD et al (2009) Pancreatic cyst fluid DNA analysis in evaluating pancreatic cysts: a report of the PANDA study. Gastrointest Endosc 69:1095-1102. https://doi.org/10.1016/j.gie.2008. 07.033
98. Shen J, Brugge WR, Dimaio CJ, Pitman MB (2009) Molecular analysis of pancreatic cyst fluid: a comparative analysis with current practice of diagnosis. Cancer 117:217-227. https://doi.org/10.1002/cncy.20027

99. Wu J, Matthaei $\mathrm{H}$, Maitra A, et al (2011) Recurrent GNAS mutations define an unexpected pathway for pancreatic cyst development. Sci Transl Med 3:92ra66. https://doi.org/10.1126/scitranslmed.3002543

100. Singhi AD, Nikiforova MN, Fasanella KE et al (2014) Preoperative GNAS and KRAS testing in the diagnosis of pancreatic mucinous cysts. Clin Cancer Res 20:4381-4389. https://doi.org/10.1158/1078-0432. CCR-14-0513

101. Al-Haddad M, Raimondo M, Woodward T et al (2007) Safety and efficacy of cytology brushings versus standard FNA in evaluating cystic lesions of the pancreas: a pilot study. Gastrointest Endosc 65:894-898. https:// doi.org/10.1016/j.gie.2006.08.047

102. Bruno M, Bosco M, Carucci P et al (2009) Preliminary experience with a new cytology brush in EUS-guided FNA. Gastrointest Endosc 70:1220-1224. https://doi.org/10.1016/j.gie.2009.05.038

103. Sendino O, Fernández-Esparrach G, Solé M et al (2010) Endoscopic ultrasonography-guided brushing increases cellular diagnosis of pancreatic cysts: a prospective study. Dig Liver Dis 42:877-881. https://doi. org/10.1016/j.dld.2010.07.009

104. El Khoury R, Kabir C, Maker VK, Banulescu M, Wasserman M, Maker AV (2018) What is the incidence of malignancy in resected intraductal papillary mucinous neoplasms? an analysis of over 100 US institutions in a single year. Ann Surg Oncol 25:1746-1751. https://doi.org/10.1245/ s10434-018-6425-6

105. Hanania AN, Bantis LE, Feng Z, et al (2016) Quantitative imaging to evaluate malignant potential of IPMNs. Oncotarget 7:85776-85784. https://doi.org/10.18632/oncotarget.11769

106. Chakraborty J, Midya A, Gazit L et al (2018) CT radiomics to predict high-risk intraductal papillary mucinous neoplasms of the pancreas. Med Phys 45:5019-5029. https://doi.org/10.1002/mp.13159

107. Attiyeh MA, Chakraborty J, Gazit L et al (2019) Preoperative risk prediction for intraductal papillary mucinous neoplasms by quantitative CT image analysis. HPB (Oxford) 21:212-218. https://doi.org/10.1016/j.hpb. 2018.07.016

108. Tobaly D, Santinha J, Sartoris R et al (2020) CT-based radiomics analysis to predict malignancy in patients with intraductal papillary mucinous neoplasm (IPMN) of the pancreas. Cancers. https://doi.org/10.3390/ cancers 12113089

109. Jeon SK, Kim JH, Yoo J, Kim JE, Park SJ, Han JK (2020) Assessment of malignant potential in intraductal papillary mucinous neoplasms of the pancreas using MR findings and texture analysis. Eur Radiol. https://doi. org/10.1007/s00330-020-07425-0

\section{Publisher's Note}

Springer Nature remains neutral with regard to jurisdictional claims in published maps and institutional affiliations.

\section{Submit your manuscript to a SpringerOpen ${ }^{\odot}$ journal and benefit from:}

- Convenient online submission

- Rigorous peer review

- Open access: articles freely available online

- High visibility within the field

- Retaining the copyright to your article

Submit your next manuscript at $\boldsymbol{\nabla}$ springeropen.com 